\title{
Mechanisms of molecular electronic rectification through electronic levels with strong vibrational coupling
}

\author{
Kuznetsov, A.M.; Ulstrup, Jens
}

Published in:

Journal of Chemical Physics

Link to article, DOI:

$10.1063 / 1.1430695$

Publication date:

2002

Document Version

Publisher's PDF, also known as Version of record

Link back to DTU Orbit

Citation (APA):

Kuznetsov, A. M., \& Ulstrup, J. (2002). Mechanisms of molecular electronic rectification through electronic levels with strong vibrational coupling. Journal of Chemical Physics, 116(5), 2149-2165.

https://doi.org/10.1063/1.1430695

\section{General rights}

Copyright and moral rights for the publications made accessible in the public portal are retained by the authors and/or other copyright owners and it is a condition of accessing publications that users recognise and abide by the legal requirements associated with these rights.

- Users may download and print one copy of any publication from the public portal for the purpose of private study or research.

- You may not further distribute the material or use it for any profit-making activity or commercial gain

- You may freely distribute the URL identifying the publication in the public portal

If you believe that this document breaches copyright please contact us providing details, and we will remove access to the work immediately and investigate your claim. 


\title{
Mechanisms of molecular electronic rectification through electronic levels with strong vibrational coupling
}

\author{
Alexander M. Kuznetsov \\ The A. N. Frumkin Institute of Electrochemistry of the Russian Academy of Sciences, Leninskij Prospect 31, \\ 117071 Moscow, Russia \\ Jens Ulstrup ${ }^{\mathrm{a})}$ \\ Department of Chemistry, Building 207, Technical University of Denmark, DK-2800 Lyngby, Denmark
}

(Received 23 April 2001; accepted 6 November 2001)

\begin{abstract}
We present a new view and an analytical formalism of electron flow through a donor-acceptor molecule inserted between a pair of metal electrodes. The donor and acceptor levels are strongly coupled to an environmental nuclear continuum. The formalism applies to molecular donoracceptor systems both in vacuum or air, and in aqueous solution under electrochemical potential control. Multifarious patterns of rectified electron flow from the negatively to the positively biased electrode arise. The electronic interaction between the donor and acceptor fragments, mutually and with the electrodes, can be weak, corresponding to the fully diabatic limit. The rectification process then reduces to a sequence of vibrationally relaxed single-electron transfer steps. In the limits where the interactions are strong, denoted as the partially and fully adiabatic limits, the character of the rectification process is different, and electron flow proceeds coherently, without vibrational relaxation. In still another class of mechanisms the electronic level broadening of either donor or acceptor from the adjacent electrode is so strong that it is comparable to the vibrational broadening. The process then reduces to a three-level transition similar to STM of large redox molecules. Recent data for rectification in hexadecyl-quinolinium tricyanodimethanide monolayers by Metzger and co-workers [J. Am. Chem. Soc. 119, 10455 (1997); Acc. Chem. Res. 32, 950 (1999)], are discussed in terms of the reported views and formalism. (C) 2002 American Institute of Physics.
\end{abstract}

[DOI: 10.1063/1.1430695]

\section{INTRODUCTION}

One of the earliest proposed principles for a molecular electronic device ${ }^{1-3}$ is the molecular rectifier, introduced by Aviram and Ratner. ${ }^{4}$ This notion represents a device, analogous to macroscopic $p-n$ semiconductor rectification, ultimately composed of molecules of the $D-S-A$ type, assembled between conducting substrates. $D$ is a donor and $A$ an acceptor group, $S$ is a rigid molecular bridge group.

Molecular donor-acceptor rectifier function can rest on the following features independently of details of the molecular mechanism. The $D-S-A$ molecules are inserted between two metallic conductors. One is the substrate on which the $D-S-A$ layer is assembled, the other one a second metallic conductor. The donor and acceptor fragment is, for example, closest to the left and right electrode, respectively (Fig. 1). In the simplest case the donor level must be below and the acceptor level above both metallic Fermi levels at zero bias voltage (see, however, Sec. VI). This ensures that the donor level is initially filled and the acceptor level vacant. On application of a positive bias voltage to the right electrode the donor and acceptor levels are both lowered but the acceptor level is shifted faster than the donor level and crosses the latter at a certain positive bias voltage. When the two levels are close, electrons will flow between the elec-

\footnotetext{
a) Author to whom correspondence should be addressed. Electronic mail: ju@kemi.dtu.dk
}

trodes via the donor-acceptor molecule. The location of the donor level below the Fermi level of the left electrode, $\varepsilon_{\mathrm{FL}}$ ensures that electron transfer (ET) from this electrode to the hole left on the donor group follows intramolecular ET from donor to acceptor. Similarly, location of the acceptor level above the Fermi level of the right electrode, $\varepsilon_{\mathrm{FR}}$, ensures that vacant levels above $\varepsilon_{\mathrm{FR}}$ can always accommodate electrons transferred from the temporarily filled acceptor level. ET in the opposite direction requires that $\varepsilon_{\mathrm{FR}}$ of the right, now electron donating electrode, crosses the acceptor level. The donor level must, further, be lifted above $\varepsilon_{\mathrm{FL}}$ of the electrode to the left. This proceeds in different potential ranges depending on the donor-acceptor level gaps, and the gaps between these levels and the Fermi levels of the electrodes. Current-bias voltage relations are therefore asymmetric, i.e., different for opposite bias voltage and current direction.

The scheme in Fig. 1 holds several other implications:

(a) Threshold, or "switchlike" effects are expected, in the sense that the bias voltage must reach a certain value before significant current flows. The current then rises to high values over a narrow bias voltage range.

(b) The intramolecular ET step follows normal patterns for molecular ET. This means that after bias voltageinduced current rise to a certain value the current passes a maximum in the current-bias voltage relation. This corresponds to transition from the normal to the 


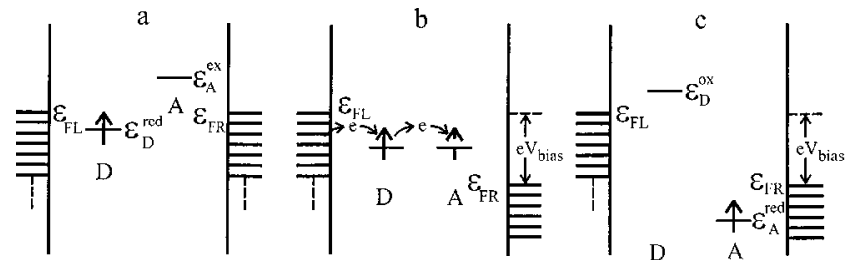

FIG. 1. Schematic view of rectified electron transfer from the electrode to the left, to the electrode to the right via a donor-acceptor molecule. The donor fragment is close to the former electrode and the acceptor fragment close to the latter. (a) Zero bias voltage. The donor level is occupied (reduced), $\varepsilon_{D}^{\text {red }}$, and below the Fermi level of the electrode to the left, $\varepsilon_{\mathrm{FL}}$. The acceptor level is vacant (oxidized), $\varepsilon_{A}^{\mathrm{ox}}$, and above the Fermi level of the electrode to the right, $\varepsilon_{\mathrm{FR}}$. (b) The electrode to the right is biased with a positive bias voltage, $V_{\text {bias }}$. The donor and acceptor levels are brought to resonance in the "energy tip region," where ET from donor to acceptor occurs. (c) Vibrational relaxation of the vacant (oxidized) donor level, $\varepsilon_{D}^{\mathrm{ox}}$, to a position above $\varepsilon_{\mathrm{FL}}$ and of the occupied (reduced) acceptor level, $\varepsilon_{A}^{\text {red }}$, to a position below $\varepsilon_{\mathrm{FR}}$. Renewed vibrational activation of $\varepsilon_{A}^{\text {red }}$ transmits the electron to vacant levels above $\varepsilon_{\mathrm{FR}}$. Negative bias of the electrode to the right increases the donor-acceptor energy gap, with insignificant current flow in the opposite direction (right to left).

activationless and inverted free energy ranges. The maximum is, however, broadened by interaction with the electrode, nuclear mode excitation, or lateral interactions in the assembly.

(c) The highest occupied (HOMO) and lowest unoccupied molecular orbitals (LUMO) of the bridge group are assumed to be off resonance with the donor and acceptor levels. These orbitals therefore assist ET or hole transfer electronically, i.e., by more favourable donoracceptor coupling or superexchange compared with vacuum.

(d) Two-level resonance between the donor and acceptor levels is broadened by two effects. One is electronic interaction between each of the levels and the nearest metallic continuum (the Fano effect). As, however, the localized donor and acceptor level populations change during electron flow, a second equally or more important broadening effect is electronic-vibrational coupling to local and collective nuclear modes in the twodimensional molecular array.

Stable current-voltage relations for electron tunneling across self-assembled organic monolayers enclosed between close to atomically planar metallic electrodes have been achieved increasingly frequently over the last few years. ${ }^{5-22}$ Different classes of organic molecules have constituted the tunnel junction, displaying strong features of rectification, switching, ${ }^{11-22}$ or pulselike behavior (negative differential resistance), ${ }^{16-19}$ cf. Sec. II. The development towards construction of actual molecular-scale devicelike systems has been paralleled by comprehensive theoretical studies of the electronic conductivity through single-molecule wires. Most of the approaches have focused on Green's function approaches to the electronic conductivity combined with electronic structure calculations of the molecular model systems. ${ }^{23-30}$ Static broadening by the nuclear motion, ${ }^{31}$ and geometry-optimization of differently charged electronic state $^{27,28}$ have also, however, been addressed. We shall return to this issue in the Discussion.

In this report we address theory and molecular mechanisms of asymmetric ET through organized $D-S-A$ arrays between a pair of metallic conductors in a different way. Emphasis is on dynamic, and strong, electronic-vibrational coupling, but electronic level broadening is also addressed. This is different from the view in Ref. 4 and from other recent theoretical approaches to molecular conductivity, ${ }^{20-31}$ but warranted by the recently discovered actual metal/ donor-acceptor-molecule/metal systems. Our approach also rests on recent broader theoretical ${ }^{32-36}$ and experimental basis $^{37-42}$ for scanning tunneling microscopy (STM) of immobilized redox molecules with low-lying redox levels both in the ex situ, vacuum or air ambient, and in situ electrochemical modes. These disclose in fact close similarities to asymmetric current flow in donor-acceptor molecules.

We first overview some systems with behavior resembling molecular rectification (Sec. II). Recent theoretical notions of STM of redox molecules are introduced in Sec. III as reference for the $D-S-A$ systems. In Sec. IV we overview molecular rectification mechanisms, with dynamic electronic-vibrational coupling as a dominating feature. The mechanisms extend to both weak and strong electronic coupling between the donor and acceptor fragments, and the metallic electrodes. Multifarious behavior arises, including sequential single-ET, vibrational coherence, superexchange, and transitions between the donor and acceptor levels, broadened by the Fano effect. In Secs. V and VI we focus on sequential and coherent ET between the metallic electrodes and the donor and acceptor fragments. This limit covers fully diabatic, and partially and fully adiabatic transitions. We provide the rate constants for the individual ET steps and show how these are related to the observable steady-state current. Current-voltage relations are analyzed in Sec. VII and are shown to display characteristic rectification and threshold effects. In Sec. VIII we address strong donor and acceptor level broadening by combined electronic-vibrational interaction and the Fano effect. This involves notions from electrochemical adsorption theory. In Sec. IX we provide a brief comparison with some recent theoretical approaches to other aspects of molecular wire conductivity, and a discussion of the recent data by Metzger ${ }^{11,12}$ in terms of the formalism.

\section{APPROACHES TOWARD MOLECULAR RECTIFYING SYSTEMS}

Attempts to construct electrically rectifying metal/D-SA/metal systems have been reported over the last decade. Geddes and associates reported Langmuir-Blodgett films of 2-bromo,5(2'-hydroxy)-tetracyanoquinodimethane dodecyloxyphenyl-carbamate. ${ }^{5}$ The current $i$ exhibited weak dependence on the bias voltage $V_{\text {bias }}$ at small $V_{\text {bias }}$, followed by a $\ln i / V_{\text {bias }}^{1 / 4}$ dependence at both positive and negative larger $V_{\text {bias }}$, and a stronger, approximately $\ln i / V_{\text {bias }}^{3}$ dependence for positive bias voltages larger than $2 \mathrm{~V}$. Martin and associates observed similar behavior of monolayer configurations of $\quad Z$ - $\beta$-(1-hexadecyl-4-quinolinium- $\alpha$-cyano-4-styryl)dicyano-methanide. ${ }^{6}$ The behavior of both systems resembles 
molecular rectification but rather similar patterns were observed for multilayer films, and views of the molecular rectifier concept would have to be modified to cope with these systems.

Other types of adsorbates display rectification at large bias voltage in STM. Aromatic thiols adsorbed on $\mathrm{Ag}(111)$ or $\mathrm{Au}(111)$ give more facile ET from sample to tip at positive bias than in the reverse direction. ${ }^{20-22,26}$ The adsorbed sulfur atom was suggested to be the donor group and the conjugate organic residue the acceptor. Nanotube devices have been shown to display rectification at particular sites along the tube, ${ }^{8}$ where local defect states were proposed as sources of conversion from metallic to discrete rectifying conductivity.

Rabe and associates reported STM spectroscopy of the peri-condensed aromatic molecule hexadedecyl hexabenzocoronen. ${ }^{9}$ This molecule forms an ordered lattice on oxidatively modified highly oriented pyrolytic graphite (HOPG). Resolution was submolecular and current-bias voltage relations for both the alkyl-substituents and the aromatic core could be recorded. Those for the former were symmetric but strong rectification was observed for the latter. This resembles a pattern for copper phthalocyanin where, however, molecular resolution was not achieved. ${ }^{10}$ The HOMO is within the bias voltage energy "tip" and likely to open resonance or multiphonon ET channels but several close-lying electronic energy levels seem to contribute.

A clear-cut case of rectification through a donoracceptor monolayer has emerged from recent studies by Metzger and co-workers. ${ }^{11,12}$ The molecule was the strongly dipolar hexadecyl-quinolinium tricyanodimethanide, enclosed between two aluminum electrodes. Monolayers of this molecule display strong rectification at room temperature and bias voltages of $\pm 1.5 \mathrm{~V}$, with conspicuous positive threshold voltages. These sharpen as the temperature is lowered to about $100 \mathrm{~K}$. In Sec. IX we shall consider this system in terms of the formalism in the present report.

An important class of self-assembled organic monolayer systems with device properties was introduced and comprehensively studied by Reed and co-workers. ${ }^{16-19}$ Currentvoltage relations of aromatic sulphides trapped between two microscopic gold electrodes showed conspicuous switching and current pulselike effects as the HOMO or LUMO was brought to resonance with the appropriate metallic Fermi level. Polyaromatic sulphides, moreover, offer the option of tuning the redox level by combinations of donor and acceptor substituents $\left(\mathrm{NH}_{2}\right.$ - and $\mathrm{NO}_{2}$-group). This gives a narrow spectroscopic feature in the form of apparently negative differential resistance, matching electronic resonances with electron transmitting states in the molecule. ${ }^{16-19,27,28} \mathrm{We}$ shall also readdress this system briefly in Sec. IX.

Other types of molecular rectification are available. Fujihira reported photoelectrochemical rectification of $D-S-A$ Langmuir films composed of porphyrins, ferrocene, and quinones. ${ }^{13,14}$ Photoexcitation brings the bridge group HOMO and LUMO close enough to the donor and acceptor levels for temporary population. Electron tunneling through intermediate single-level states is also the basis for configurable rotaxane-based molecular switches. ${ }^{15}$ These can be "open" or "closed" by holding the redox active switch in the oxidized and reduced state, respectively. Arrays of devices could be configured to molecule-based logic AND and OR gates. Such intermediate single-level resonance tunneling is most suitably addressed by ET theory of three-level systems with dynamically populated intermediate states $^{32-34,36,43,44}$ which has also become a theoretical frame for STM of redox molecules. ${ }^{32-35}$ STM of electronically resonating molecules has some resemblance to the molecular rectifier but is simpler by having only a single level between the two electrodes. As a reference we therefore first provide some recent observations in the theory of in situ STM of redox molecules.

\section{MOLECULAR RECTIFICATION AND IN SITU STM OF REDOX MOLECULES}

STM of redox-active adsorbates with low-lying populated intermediate states have been reported in both the ex situ ${ }^{42,45,46}$ and electrochemical in situ modes. ${ }^{37-40,47-49}$ Spectroscopic current-voltage features have been found only in a few cases ${ }^{36-39,42,45}$ but hold perspectives for distinction between different tunnelling mechanisms and for devicelike systems such as rectifiers or memory storage elements. STM of redox adsorbates has been given theoretical frames ${ }^{32-34,50,51}$ based on ET theory, ${ }^{52-54}$ with the following observations:

(1) Electron tunneling through molecular redox levels invariably involves electronic-vibrational interaction. This leads to different vibrationally assisted, multiphonon tunneling mechanisms known as resonance, coherent, and sequential tunneling. ${ }^{36,44}$

(2) Electronic-vibrational interactions have two effects in particular. One is that the intermediate state equilibrium energies in the oxidized (vacant) and reduced (populated) states are different (Figs. 1 and 2). The second is that fluctuations in the nuclear coordinates are crucial by inducing resonance with the Fermi levels of the enclosing metallic substrate and tip.

(3) The most important controlling factor is the bias voltage but a second, electrochemical potential difference is crucial for in situ STM, namely, the substrate-solution potential difference. ${ }^{55-57}$ This double potentiostatic control also offers two spectroscopic correlations, i.e., currentbias voltage relations at given overpotential, and current-overvoltage relations where both the substrate and tip potentials are varied in parallel.

(4) The observations in (1)-(3) also apply broadly to molecular rectification with two local (donor and acceptor) redox centers.

Tunneling through adsorbed redox molecules in electrochemical in situ STM can follow patterns of resonance, co- 

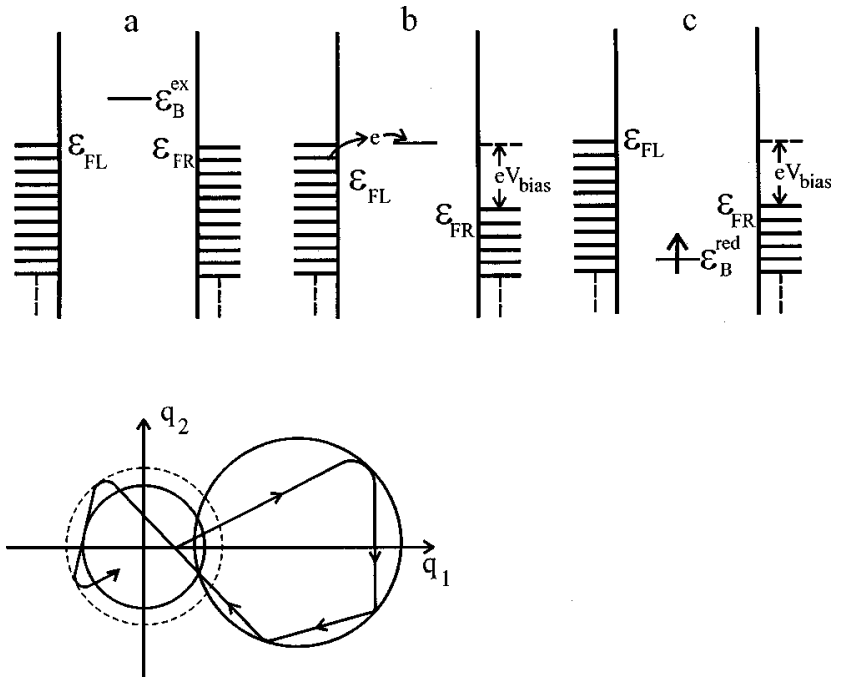

FIG. 2. Electronic level configurations in STM of a redox molecule. Top: (a) the redox level $\varepsilon_{B}^{\mathrm{ox}}$ is vacant (oxidized) and above the Fermi levels of the substrate, $\varepsilon_{\mathrm{FL}}$, and tip, $\varepsilon_{\mathrm{FR}}$, at zero bias voltage. This configuration also represents in situ STM at fixed overvoltage. (b) A positive bias voltage on the tip, $V_{\text {bias }}$, takes $\varepsilon_{B}^{\text {ox }}$ close to resonance with populated levels around $\varepsilon_{\mathrm{FL}}$ for ET to the redox level. (c) The reduced redox level, $\varepsilon_{B}^{\text {red }}$, relaxes to a position below $\varepsilon_{\mathrm{FR}}$. Renewed thermal activation transmits the electron from $\varepsilon_{B}^{\text {red }}$ to the tip. Bottom: two-mode potential surfaces of the process at the top, viewed from the energy axis. The initial state is the small cross section to the left. The cross section to the right is the relaxed intermediate state corresponding to configuration (c) (top). The cross section indicated by dashed lines is the final state where the electron has been transmitted to the tip. The arrow indicates one of many trajectories in the two-step transition from the initial to the final state.

herent two-step, and sequential two-step ET. Two-step ET is the mechanism appropriate to the rectifier and follows three kinds of behavior:

(i) The transitions between the redox level and each of the electrodes can belong to the adiabatic limit of strong interaction. Tunneling features are then reflected in the tunneling current, and by the number of electronic levels engaged in the transition, but the current-voltage relations are dominated by the nuclear activation energy and the bias and overpotential dependence of this quantity. The strong molecule-electrode interactions leads each cycle of (iii)

(ii) Level interaction with one electrode can be strong and weak with the other electrode, so that the former belongs to the adiabatic, the latter to the diabatic limit. Diabatic tunneling features are strongly reflected in the current while fast equilibrium is established at the adiabatic contact.

Both interactions can be weak and the transitions fully diabatic. Tunneling features of both transitions then appear in the current, which reduces to two sequential diabatic single-ET steps.

These distinctions also apply to the molecular rectifier, which involves an additional adiabatic or diabatic intramolecular ET step. With a view on the rectifier the following observations from in situ STM (Refs. 32-36, 58) are useful:

Spectroscopic current-voltage features are expected when the bias voltage is smaller than the environmental reorganization Gibbs free energy, $E_{r}$, i.e., $\left|\mathrm{eV}_{\text {bias }}\right|$ $<E_{r} . E_{r}$ is significantly smaller in the tunnel gap, say $E_{r} \approx 0.2 \mathrm{eV}$, than in the semi-infinite configuration of electrochemical ET. ${ }^{33}$ The features are characteristic resonances in the current- and derivative currentvoltage relations. The position and width reflect electronic-vibrational coupling (the reorganization Gibbs free energy), the potential distribution in the tunnel gap, and interactions with the metallic electrodes.

(II) The current-overvoltage relations change to asymmetric form at large $V_{\text {bias }}$. The current rises initially, followed by current independence of $\eta$ over a broad range $\left(\eta \rightarrow V_{\text {bias }}\right){ }^{36,58}$ The current-overpotential relation is also asymmetric in the sense that the overpotential ranges of current rise and constant current are different in the two current directions. ET through single intermediate states can therefore also display rectifying effects.
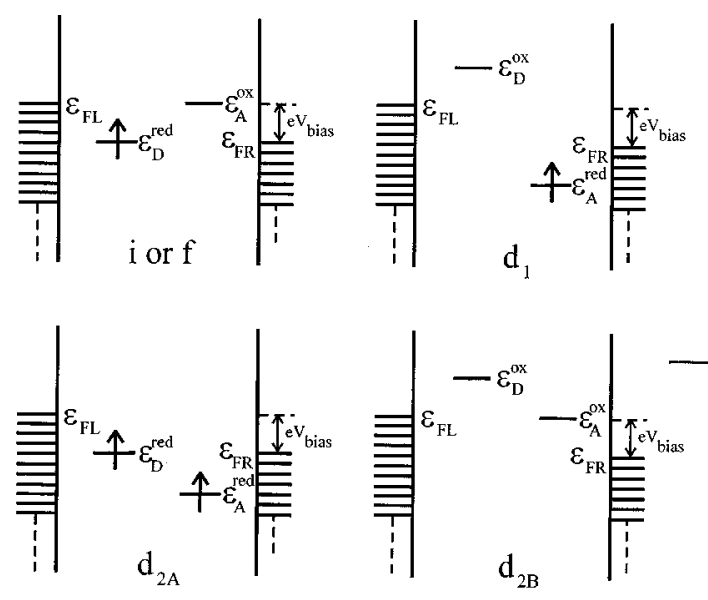

FIG. 3. Level configurations in electron flow through a donor-acceptor molecule. Left: electronic levels in the initial $(i)$, final $(f)$, first intermediate $\left(d_{1}\right)$, and two second intermediate states $\left(d_{2 A}\right.$ and $\left.d_{2 B}\right)$. Oxidized and reduced donor and acceptor levels shown. Right: potential surface cross sections of the four states viewed as in Fig. 2. The arrows show two trajectories $i \rightarrow d_{1} \rightarrow d_{2 \mathrm{~A}}$ $\rightarrow f$, and $i \rightarrow d_{1} \rightarrow d_{2 \mathrm{~B}} \rightarrow f$ from the initial to the final state. 


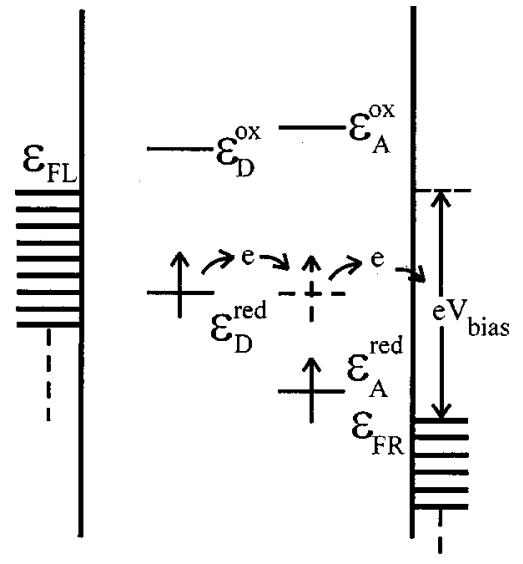

FIG. 4. Electron flow through the donor-acceptor molecule at large bias voltage. Both the oxidized, $\varepsilon_{A}^{\text {ox }}$, and the reduced acceptor level, $\varepsilon_{A}^{\text {red }}$, are above the Fermi level of the positively biased electrode, $\varepsilon_{\mathrm{FR}}$. The reduced acceptor level is dynamically populated, without vibrational relaxation, and two electrons are transferred in a single reactive event.

\section{MECHANISMS OF ELECTRONIC RECTIFICATION THROUGH A DONOR-ACCEPTOR MOLECULE}

Figure 1 represents the simplest scheme of molecular rectification via separate donor and acceptor fragments. Rectification can proceed by the following different mechanisms, rooted in different electronic couplings between the donor and acceptor fragments and the electrodes:

(A) The sequence of rectifying steps can constitute separate electronic transitions, from the negatively to the positively biased electrode via the donor and acceptor fragments. Each transition proceeds through a vibrationally relaxed intermediate state, but the order of ET events is different in different bias voltage ranges. The ET sequence involves several intermediate electronic states. The initial and final states represent the same electronic population except that the electronic levels are shifted by the bias voltage. The first intermediate state in the scheme in Fig. 1 is always present and represented by a vacant donor and an occupied acceptor level. This is followed by a second intermediate state where the donor and acceptor levels are either both occupied or vacant [Fig. 3 (left)]. Figure 3 (right) shows the potential surface configurations, which can be compared to those for in situ STM (Fig. 2).

(B) For the rectifier to be efficient the current should not be limited by tunneling between the donor and acceptor and their adjacent electrodes. The adiabatic limit of strong interaction at the metal-donor and acceptormetal contacts is therefore appropriate. The diabatic limit of weak interactions is, however, conceptually the simplest and will be considered first. Both limits of intramolecular ET are appropriate and will be denoted as the fully and partially adiabatic limits when the metal-donor and acceptor-metal transitions remain fully adiabatic.

(C) The character of electron flow through charge-relaying intermediate states may change at certain bias voltages. When the $V_{\text {bias }}$ is large and positive (the Fermi level of

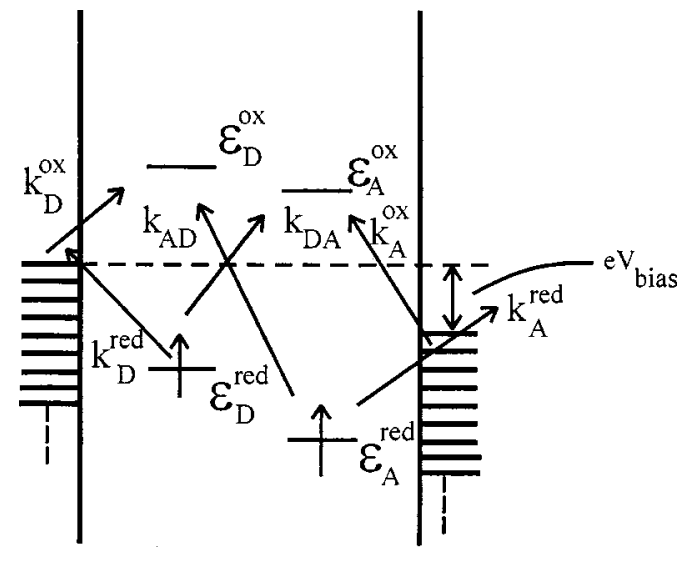

FIG. 5. Scheme of interfacial and intramolecular ET reactions for combinations of vacant and occupied donor and acceptor levels in the initial state.

the electrode to the right, $\varepsilon_{\mathrm{FR}}$, below the Fermi level of the electrode to the left, $\varepsilon_{\mathrm{FL}}$ ), the acceptor level in the equilibrated populated state may be shifted to a position above $\varepsilon_{\mathrm{FR}}$. ET from donor to acceptor then proceeds through a dynamically populated $D^{+} A^{-}$state. This notion implies that there is no vibrational relaxation in this state prior to subsequent fast ET from the populated acceptor to vacant levels above $\varepsilon_{\mathrm{FR}} \cdot{ }^{34,36,44,58}$ This mechanism is illustrated in Fig. 4. A similar view applies when the bias voltage is large and negative, and the equilibrated oxidized acceptor level is below $\varepsilon_{\mathrm{FR}}$ of the negatively biased (right) electrode but above $\varepsilon_{\mathrm{FL}}$ of the electrode to the left.

(D) Other level configurations where donor and acceptor levels are both vacant or both occupied at zero bias can be envisaged (Fig. 5). These will be considered in Sec. VIB.

(E) Either the donor or the acceptor level, or both may be strongly broadened by electronic interaction with the metallic surfaces (Fig. 6). The rectifying process resembles STM of a redox molecule with a single molecular level when only one of the levels is broadened The current then follows closely the pattern from STM (Refs. 32-34) but involves convolution of the electronic broadening with the vibrational continuum. A two-level limit arises when both donor and acceptor

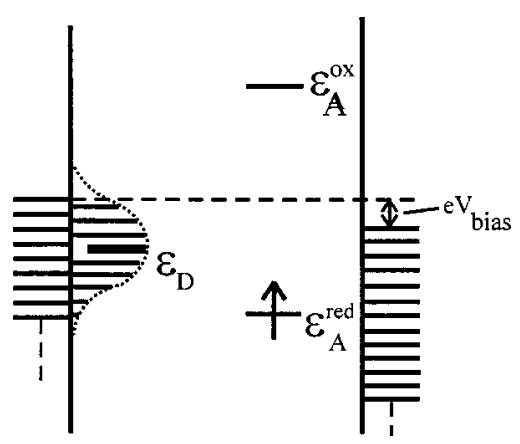

FIG. 6. Molecular rectification when the electronic interaction between the donor level, $\varepsilon_{D}$, below $\varepsilon_{\mathrm{FL}}$, is strong and electronic broadening comparable to vibrational broadening. The broadened donor level density and the discrete oxidized, $\varepsilon_{A}^{\text {ox }}$, and reduced acceptor level, $\varepsilon_{A}^{\text {red }}$, are also shown. 
levels are broadened by strong electronic surface interactions convoluted with the vibrational modes.

\section{RECTIFICATION BY FULLY DIABATIC SEQUENTIAL ET}

We address here the case when all interfacial and intramolecular ET steps belong to the fully diabatic limit.

\section{A. Rectification by stepwise transitions in sequential diabatic ET}

We confine the analysis to the case where $V_{\text {bias }}$, is small compared to the electronic-vibrational coupling (Fig. 1). This implies that subsequent to ET from the donor to the acceptor the former level is shifted vertically from a position below the Fermi level of the electrode to the left, $\varepsilon_{\mathrm{FL}}$, to a position above $\varepsilon_{\mathrm{FL}}$. The acceptor level is, similarly, shifted from a position above the Fermi level of the electrode to the right, $\varepsilon_{\mathrm{FR}}$, to a position below $\varepsilon_{\mathrm{FR}}$. Bias voltage and all electronic levels are counted from $\varepsilon_{\mathrm{FL}}$. The scheme for stepwise ET from the left to the right electrode, rectified by ET through the donor-acceptor molecule is then

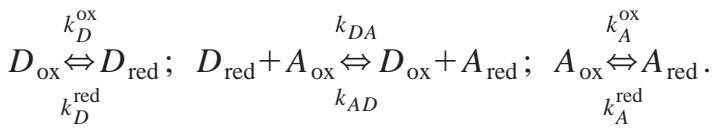

$D_{\text {ox }}$ and $D_{\text {red }}$ are the oxidized (vacant) and reduced (populated) donor, respectively, $A_{\text {ox }}$ and $A_{\text {red }}$ the analogous forms of the acceptor. $k_{S}^{\text {ox }}$ and $k_{S}^{\text {red }}, S=D, A$, are the rate constants $\left(\mathrm{s}^{-1}\right)$ of the interfacial ET processes in the first and the third equation in Eq. (1), $k_{D A}$ and $k_{A D}$ the rate constants for forward and reverse intramolecular ET, respectively (Fig. 1).

We introduce $P_{D}$ and $P_{A}$ as the probability that the donor and acceptor level, respectively, is populated. The steady-state rectified current is then given by

$$
\begin{aligned}
& -k_{D}^{\mathrm{ox}}\left(1-P_{D}\right)+k_{D}^{\mathrm{red}} P_{D}+k_{D A} P_{D}\left(1-P_{A}\right) \\
& -k_{A D}\left(1-P_{D}\right) P_{A}=0, \\
& -k_{D A} P_{D}\left(1-P_{A}\right)+k_{A D}\left(1-P_{D}\right) P_{A} \\
& +k_{A}^{\mathrm{red}} P_{A}-k_{A}^{\mathrm{ox}}\left(1-P_{A}\right)=0 .
\end{aligned}
$$

Adding Eq. (3) to Eq. (2) we obtain

$$
-k_{D}^{\mathrm{ox}}\left(1-P_{D}\right)+k_{D}^{\mathrm{red}} P_{D}+k_{A}^{\mathrm{red}} P_{A}-k_{A}^{\mathrm{ox}}\left(1-P_{A}\right)=0,
$$

or the following relation between $P_{A}$ and $P_{D}$ :

$$
P_{A}=\frac{k_{A}^{\mathrm{ox}}+k_{D}^{\mathrm{ox}}-\left(k_{D}^{\mathrm{ox}}+k_{D}^{\mathrm{red}}\right) P_{D}}{k_{A}^{\mathrm{ox}}+k_{A}^{\mathrm{red}}} .
$$

Insertion of Eq. (5) into Eq. (2) gives, after rearranging terms,

$$
\begin{aligned}
& P_{D}^{2} \frac{\left(k_{D A}-k_{A D}\right)\left(k_{D}^{\mathrm{ox}}+k_{D}^{\mathrm{red}}\right)}{k_{A}^{\mathrm{ox}}+k_{A}^{\mathrm{red}}} \\
& \quad+P_{D}\left\{\frac{k_{A D}\left(k_{D}^{\mathrm{ox}}+k_{D}^{\mathrm{red}}\right)-\left(k_{D A}-k_{A D}\right)\left(k_{A}^{\mathrm{ox}}+k_{D}^{\mathrm{ox}}\right)}{k_{A}^{\mathrm{ox}}+k_{D}^{\mathrm{ox}}}+k_{D}^{\mathrm{ox}}\right. \\
& \left.\quad+k_{D}^{\mathrm{red}}+k_{D A}\right\}-k_{D}^{\mathrm{ox}}-\frac{k_{A D}\left(k_{A}^{\mathrm{ox}}+k_{D}^{\mathrm{ox}}\right)}{k_{A}^{\mathrm{ox}}+k_{A}^{\mathrm{red}}}=0
\end{aligned}
$$

or

$$
\begin{aligned}
P_{D}^{2}\{ & \left.\left(k_{D}^{\mathrm{ox}}+k_{D}^{\mathrm{red}}\left(k_{D A}-k_{A D}\right)\right)\right\}+P_{D}\left\{\left(k_{A}^{\mathrm{ox}}+k_{A}^{\mathrm{red}}\right)\left(k_{D}^{\mathrm{ox}}+k_{D}^{\mathrm{red}}\right)\right. \\
& +k_{D A}\left(k_{A}^{\mathrm{ox}}+k_{A}^{\mathrm{red}}\right)+k_{A D}\left(k_{D}^{\mathrm{ox}}+k_{D}^{\mathrm{red}}\right)-\left(k_{D A}-k_{A D}\right) \\
& \left.\times\left(k_{A}^{\mathrm{ox}}+k_{D}^{\mathrm{ox}}\right)\right\}-\left\{k_{D}^{\mathrm{ox}}\left(k_{A}^{\mathrm{ox}}+k_{A}^{\mathrm{red}}\right)+k_{A D}\left(k_{A}^{\mathrm{ox}}+k_{D}^{\mathrm{ox}}\right)\right\}=0 .
\end{aligned}
$$

Equation (7) has a single solution for $P_{D}$, i.e.,

$$
\begin{aligned}
P_{D}= & \frac{1}{2\left(k_{D}^{\mathrm{ox}}+k_{D}^{\mathrm{red}}\right)\left(k_{D A}-k_{A D}\right)}\left\{\left(k_{D A}-k_{A D}\right)\left(k_{A}^{\mathrm{ox}}+k_{D}^{\mathrm{ox}}\right)-\left(k_{A}^{\mathrm{ox}}\right.\right. \\
& \left.+k_{A}^{\mathrm{red}}\right)\left(k_{D}^{\mathrm{ox}}+k_{D}^{\mathrm{red}}\right)-k_{D A}\left(k_{A}^{\mathrm{ox}}+k_{A}^{\mathrm{red}}\right)-k_{A D}\left(k_{D}^{\mathrm{ox}}+k_{D}^{\mathrm{red}}\right) \\
& +\left\{\left[\left(k_{D A}-k_{A D}\right)\left(k_{A}^{\mathrm{ox}}+k_{D}^{\mathrm{ox}}\right)-\left(k_{A}^{\mathrm{ox}}+k_{A}^{\mathrm{red}}\right)\left(k_{D}^{\mathrm{ox}}+k_{D}^{\mathrm{red}}\right)\right.\right. \\
& \left.-k_{D A}\left(k_{A}^{\mathrm{ox}}+k_{A}^{\mathrm{red}}\right)-k_{A D}\left(k_{D}^{\mathrm{ox}}+k_{D}^{\mathrm{red}}\right)\right]^{2}+4\left(k_{D}^{\mathrm{ox}}+k_{D}^{\mathrm{red}}\right)\left(k_{D A}\right. \\
& \left.\left.\left.-k_{A D}\right)\left[k_{D}^{\mathrm{ox}}\left(k_{A}^{\mathrm{ox}}+k_{A}^{\mathrm{red}}\right)+k_{A D}\left(k_{A}^{\mathrm{ox}}+k_{D}^{\mathrm{ox}}\right)\right]\right\}^{1 / 2}\right\} .
\end{aligned}
$$

Equations (5) and (8) are thus the steady-state electronic populations of the donor and acceptor fragments. $P_{D}$ and $P_{A}$ give the observable steady-state rectifier current,

$$
\begin{aligned}
i & =e C\left\{k_{D}^{\mathrm{ox}}\left(1-P_{D}\right)-k_{D}^{\mathrm{red}} P_{D}\right\} \\
& =e C\left\{k_{D}^{\mathrm{ox}}-\left(k_{D}^{\mathrm{ox}}+k_{D}^{\mathrm{red}}\right) P_{D}\right\},
\end{aligned}
$$

where $C$ either represents a single molecule $(C=1)$, or the donor-acceptor molecular coverage in the gap between the electrodes. Equations (5), (8), and (9) are general for diabatic electronic transitions and small bias voltage. The character of the rectification process is different in the partially and fully adiabatic limits (Sec. VI).

\section{B. ET rate constants}

The level populations $P_{D}$ and $P_{A}$ [Eq. (9)] are determined by the diabatic rate constants of sequential interfacial and intramolecular ET steps via Eqs. (5)-(8).

We shall use the following broadly valid interfacial ET rate constant forms: $:^{53,54}$ 


$$
\begin{aligned}
k_{D}^{\mathrm{ox}}= & \kappa_{L} \rho_{L} \frac{2 k_{B} T}{\alpha_{L}} \frac{\omega_{\mathrm{eff}}}{2 \pi} \exp \left[-\frac{\left(E_{r}^{D}-e \xi_{D} \eta-e \gamma_{D} V_{\text {bias }}\right)^{2}}{4 E_{r}^{D} k_{B} T}\right], \\
k_{A}^{\mathrm{ox}}= & \kappa_{R} \rho_{R} \frac{2 k_{B} T}{\alpha_{R}} \frac{\omega_{\text {eff }}}{2 \pi} \\
& \times \exp \left[-\frac{\left(E_{r}^{A}+e V_{\text {bias }}-e \xi_{A} \eta-e \gamma_{A} V_{\text {bias }}\right)^{2}}{4 E_{r}^{A} k_{B} T}\right],
\end{aligned}
$$

$$
\begin{aligned}
& k_{D}^{\mathrm{red}}=k_{D}^{\mathrm{ox}} \exp \left(-\frac{e \xi_{D} \eta+e \gamma_{D} V_{\text {bias }}}{k_{B} T}\right), \\
& k_{A}^{\mathrm{red}}=k_{A}^{\mathrm{ox}} \exp \left(\frac{e V_{\text {bias }}-e \xi_{A} \eta-e \gamma_{A} V_{\text {bias }}}{k_{B} T}\right),
\end{aligned}
$$

where $\alpha_{L}$ and $\alpha_{R}$ are the transfer coefficients for interfacial ET at the left and right metal, respectively. The intramolecular ET rate constants are

$$
k_{D A}=\kappa_{D A} \frac{\omega_{\text {eff }}}{2 \pi} \exp \left[-\frac{\left(E_{r}^{D}+E_{r}^{A}+e \xi_{D} \eta+e \gamma_{D} V_{\text {bias }}-e \xi_{A} \eta-e \gamma_{A} V_{\text {bias }}\right)^{2}}{4\left(E_{r}^{D}+E_{r}^{A}\right) k_{B} T}\right],
$$

$$
k_{A D}=k_{D A} \exp \left(\frac{e \xi_{D} \eta+e \gamma_{D} V_{\text {bias }}-e \xi_{A} \eta-e \gamma_{A} V_{\text {bias }}}{k_{B} T}\right) .
$$

The origin and validity conditions of these forms are discussed elsewhere. ${ }^{32-34,36,53,54}$ We note:

(A) Equations (10) -(13) apply to rectification in a continuous, aqueous or other condensed matter environment where both electrodes are subject to electrochemical potential control. Two potential differences appear, namely the bias voltage, $V_{\text {bias }}$, and the overvoltage relative to a reference electrode, $\eta$. Similar forms apply to vacuum or air when the electronic coupling to intramolecular or collective modes is strong, except that the $\eta$-dependent terms are absent.

(B) The activation free energies contain: (i) The reorganization Gibbs free energies for oxidation or reduction of the donor and acceptor, $E_{r}^{D}$ and $E_{r}^{A}$, respectively. For the sake of simplicity the reorganization free energy of the intramolecular ET step is taken as the sum of $E_{r}^{D}$ and $E_{r}^{A}$. (ii) The fraction of the total potential drop between the electrode to the left and the bulk solution, at the site of the donor fragment, $\xi_{D} \cdot \xi_{A}$ has a similar meaning for the acceptor. (iii) The fractions of the bias potential drops at the site of the donor and acceptor, $\gamma_{D}$ and $\gamma_{A}$, respectively.

(C) $\omega_{\text {eff }}$ is the effective vibrational frequency of the nuclear modes reorganized, $\rho_{L}$ and $\rho_{R}$ the electronic energy densities of states of the electrode to the left and right, respectively. $\kappa_{L}$ and $\kappa_{R}$ are the transmission coefficients for interfacial ET, and $\kappa_{D A}$ the transmission coefficient for intramolecular ET.

(D) Equations (10)-(12) apply explicitly to symmetric locations of the energy levels $\varepsilon_{D}^{\text {ox }}$ and $\varepsilon_{D}^{\text {red }}$, with respect to the Fermi energy $\varepsilon_{\mathrm{FL}}$ and of the levels $\varepsilon_{A}^{\mathrm{ox}}$ and $\varepsilon_{A}^{\text {red }}$ with respect to $\varepsilon_{\mathrm{FR}} \cdot e \xi_{D} \eta$ and $e \xi_{A} \eta$ in Eqs. (10)-(12) and all subsequent equations must be replaced by $e \xi_{D} \eta+\varepsilon_{D 0}$ and $e \xi_{A} \eta+\varepsilon_{A 0}$, respectively, if the levels are shifted by $\varepsilon_{D 0}$ and $\varepsilon_{A 0}$ at equilibrium, where $\varepsilon_{D 0}$ $=\varepsilon_{D}^{\mathrm{red}}-E_{r}^{D}$ and $\varepsilon_{A 0}=\varepsilon_{A}^{\mathrm{red}}-E_{r}^{A}$, i.e.,

$$
e \xi_{D} \eta \rightarrow e \xi_{D} \eta+\varepsilon_{D 0} ; e \xi_{A} \eta \rightarrow e \xi_{A} \eta+\varepsilon_{A 0} .
$$

Equations (5)-(9) together with the rate constants in Eqs. (10)-(14) were derived as the rectified electron flow from left to right (Figs. 1 and 3) under the influence of a positive bias voltage smaller than the total reorganization Gibbs free energy. The same formalism applies, however, for current flow in the opposite direction, at negative bias voltage.

\section{FULLY AND PARTIALLY ADIABATIC TRANSITIONS}

Both interfacial ET steps are adiabatic and electronically facile in these limits. This changes the character of the processes drastically compared with the fully diabatic limit.

\section{A. Dynamically populated intermediate electronic states in adiabatic transitions}

As for STM of single-level redox molecules, the physical nature of the transitions in electronic rectification is different when the molecular interactions with the metal electrodes is strong (Fig. 4). Very similar patterns apply for the fully and partially adiabatic limits, where the intramolecular electronic coupling is strong and weak, respectively, while the interfacial couplings remain strong in both cases. The transmission coefficient, $\kappa_{D A} \ll 1$ appears in the rate constant of this step in the partially adiabatic cases while $\kappa_{D A} \rightarrow 1$ in the fully adiabatic limit.

When the donor level is initially populated and the acceptor level vacant (Fig. 4), nuclear configurational fluctuations raise the populated donor level and lower the vacant acceptor level to resonance at an energy above the Fermi level of the positively biased electrode. Subsequent to intramolecular ET, $D-S-A \rightarrow D^{+}-S-A^{-}$, relaxation of the, now occupied, acceptor level towards lower energy follows. Due to the strong electronic interaction at the metallic contacts this is, however, intercepted by fast interfacial ET from the occupied acceptor level to the positively biased electrode and fast ET from the negatively biased electrode to the empty donor level. This is the first distinction from the fully diabatic limit, i.e., the intermediate $D^{+}-S-A^{-}$state is dynamically populated, with only partial vibrational relaxation. 
The two interfacial ET steps reverse the electronicvibrational relaxation. The reoccupied but unrelaxed donor level initiates a second trajectory downwards and the vacant unrelaxed acceptor level a second trajectory upwards. As a result the levels cross a second time and a second electron is transferred. Two electrons are thus transferred in a single cycle. This is the second distinction from the fully diabatic limit.

\section{B. A formalism for rectification in the fully and partially adiabatic limits}

We provide first the current rectification in the configuration $D-S-A$ (Figs. 1 and 5). We consider positive and negative bias voltages separately.

\section{Rate constants at positive bias voltages}

The process is based on the following set of potential energies around the donor and acceptor group in the oxidized ("ox") and reduced ("red") states,

$$
\begin{aligned}
& U_{D}^{\mathrm{ox}}=\frac{1}{2} \hbar \omega q_{D}^{2}+\varepsilon_{D}^{\mathrm{ox}}, U_{A}^{\mathrm{ox}}=\frac{1}{2} \hbar \omega q_{A}^{2}+\varepsilon_{A}^{\mathrm{ox}}, \\
& U_{D}^{\mathrm{red}}=\frac{1}{2} \hbar \omega q_{D}^{2}-g_{D} q_{D}-e \xi_{D} \eta-e \gamma_{D} V_{\mathrm{bias}}+\varepsilon_{D}^{\mathrm{ox}}, \\
& U_{A}^{\mathrm{red}}=\frac{1}{2} \hbar \omega q_{A}^{2}-g_{A} q_{A}-e \xi_{A} \eta-e \gamma_{A} V_{\text {bias }}+\varepsilon_{A}^{\mathrm{ox}} .
\end{aligned}
$$

The initial and final state potential surfaces are

$$
U_{i}\left(q_{D}, q_{A}\right)=U_{D}^{\mathrm{red}}+U_{A}^{\mathrm{ox}}, U_{f}\left(q_{D}, q_{A}\right)=U_{D}^{\mathrm{ox}}+U_{A}^{\mathrm{red}} .
$$

$q_{D}, q_{A}$ is a set of harmonic local nuclear coordinates with the frequency $\omega, \varepsilon_{D}^{\mathrm{ox}}$, and $\varepsilon_{A}^{\mathrm{ox}}$ the energies of the vacant electronic level of the donor and acceptor, respectively, and $g_{D}$ and $g_{A}$ electronic-vibrational coupling factors at the donor and acceptor site. $g_{D}$ and $g_{A}$ are related to the equilibrium nuclear displacements in the reduced states, $q_{D 0}$ and $q_{A 0}$, and the reorganization energies, $E_{r}^{D}$ and $E_{r}^{A}$, by

$$
\begin{aligned}
& q_{D 0}=\frac{g_{D}}{\hbar \omega}, \quad E_{r}^{D}=\frac{1}{2} \frac{g_{D}^{2}}{\hbar \omega}=\frac{1}{2} \hbar \omega q_{D 0}^{2}, \\
& q_{A 0}=\frac{g_{A}}{\hbar \omega}, \quad E_{r}^{A}=\frac{1}{2} \frac{g_{A}^{2}}{\hbar \omega}=\frac{1}{2} \hbar \omega q_{A 0}^{2} .
\end{aligned}
$$

The process $D-S-A \rightarrow D^{+}-S-A^{-}$is induced by energy resonance at the saddle point of the potential surface crossing, $q_{D}^{*}, q_{A}^{*}$, where $U_{i}=U_{f}$, or

$$
\begin{aligned}
\varepsilon_{D}^{\mathrm{ox}}- & e \xi_{D} \eta-e \gamma_{D} V_{\mathrm{bias}}-g_{D} q_{D}^{*} \\
& =\varepsilon_{A}^{\mathrm{ox}}-e \xi_{A} \eta-e \gamma_{A} V_{\mathrm{bias}}-g_{A} q_{A}^{*}
\end{aligned}
$$

subject to

$$
\frac{d U_{D}^{\mathrm{red}}}{d q_{D}}=\hbar \omega q_{A}^{*} \frac{g_{D}}{g_{A}}+\hbar \omega\left(q_{D}^{*}-q_{D 0}\right)=0,
$$

since $d q_{A} / d q_{D}=g_{D} / g_{A}$. Provided that the resonating occupied donor level and vacant acceptor level remain between the two Fermi levels, i.e.,

$$
\begin{aligned}
& \varepsilon_{D}^{\mathrm{red}}\left(q_{D}^{*}\right)<\varepsilon_{\mathrm{FL}} \quad \text { and } \quad \varepsilon_{A}^{\mathrm{ox}}\left(q_{A}^{*}\right)>\varepsilon_{\mathrm{FR}}, \text { or } \\
& \varepsilon_{\mathrm{FR}} \leqslant \varepsilon_{D}^{\mathrm{ox}}-e \xi_{D} \eta-e \gamma_{D} V_{\text {bias }}-g_{D} q_{D}^{*} \leqslant \varepsilon_{\mathrm{FL}},
\end{aligned}
$$

Eqs. (18)-(20) give for the saddle point coordinates,

$$
\begin{aligned}
& q_{D}^{*}=\frac{1}{1+\frac{E_{r}^{D}}{E_{r}^{A}}}\left\{\sqrt{\frac{2 E_{r}^{D}}{\hbar \omega}}+\frac{\sqrt{2 E_{r}^{D} \hbar \omega}}{2 E_{r}^{A} \hbar \omega}\left[\varepsilon_{D}^{\mathrm{ox}}-\varepsilon_{A}^{\mathrm{ox}}+e \xi_{A} \eta\right.\right. \\
& \left.\left.+e \gamma_{A} V_{\text {bias }}-e \xi_{D} \eta-e \gamma_{D} V_{\text {bias }}\right]\right\}, \\
& q_{A}^{*}=\sqrt{\frac{E_{r}^{D}}{E_{r}^{A}}} q_{D}^{*}+\frac{1}{\sqrt{2 E_{r}^{A} \hbar \omega}}\left[\varepsilon_{A}^{\mathrm{ox}}-\varepsilon_{D}^{\mathrm{ox}}+e \xi_{D} \eta+e \gamma_{D} V_{\text {bias }}\right. \\
& \left.-e \xi_{A} \eta-e \gamma_{A} V_{\text {bias }}\right] \text {. }
\end{aligned}
$$

The donor and acceptor energy at resonance is, at positive bias voltage,

$$
\begin{aligned}
-e V_{\text {bias }} & <\varepsilon^{*}-\varepsilon_{\mathrm{FL}} \\
& =-\frac{\left(e \xi_{A} \eta+e \gamma_{A} V_{\mathrm{bias}}\right) E_{r}^{D}+\left(e \xi_{D} \eta+e \gamma_{D} V_{\text {bias }}\right)}{E_{r}^{D}+E_{r}^{A}} \\
& <0 .
\end{aligned}
$$

The calculation of the rate constant for the intramolecular ET process $D-S-A \rightarrow D^{+}-S-A^{-}$follows the procedure in Appendix $A$. The result is

$$
\begin{aligned}
& k_{D A}^{r / o}=2 \kappa_{D A} \frac{\omega}{2 \pi} \tanh \frac{e\left|V_{\text {bias }}\right|}{\Delta} \cdot \exp \left\{-\frac{\left[E_{r}^{A}+E_{r}^{A}+e \xi_{D} \eta+e \gamma_{D} V_{\text {bias }}-e \xi_{A} \eta-e \gamma_{A} V_{\text {bias }}\right]^{2}}{4 E_{r}^{A} k_{B} T}\right\}, \\
& \Delta=\sqrt{\frac{4 E_{r}^{D} E_{r}^{A} k_{B} T}{E_{r}^{D}+E_{r}^{A}}} .
\end{aligned}
$$

The observable current from the negatively to the positively biased electrode is, finally

$$
i=e C k_{D A}^{r / o} \text {. }
$$

As noted, the factor 2 in the pre-exponential part of Eq. (23) accounts for the twofold level crossing. The hyperbolic tangent has been introduced as an interpolation function. $\Delta$ is the thermal broadening in the $q_{D}$ and $q_{A}$ modes (Appendix A). If this width is small compared with $e V_{\text {bias }}$, the rate constant reduces to the form for intramolecular ET between 
the donor and acceptor. If $\Delta$ is large compared with $e V_{\text {bias }}$ only the fraction $e V_{\text {bias }} / \Delta$ contributes. Equations (23) and (24) apply in both adiabatic and diabatic limits of intramolecular ET, with $\kappa_{D A} \rightarrow 1$ and $\kappa_{D A} \ll 1$, respectively.

The other configurations shown in Fig. 5 follow similar lines (Appendix B):

a. Initial configuration $D^{+}-S-A^{-}$: The $D$-level is initially vacant and above $\varepsilon_{\mathrm{FL}}$, and the acceptor level occupied and below $\varepsilon_{\mathrm{FR}}$. Thermal fluctuations induce level shifts in opposite directions, with intramolecular ET in the "energy tip" region where the donor level becomes occupied and the acceptor level vacant. The rate constant is

$$
\begin{aligned}
k_{D A}^{o / r}= & 2 \kappa_{D A} \frac{\omega}{2 \pi} \sqrt{\frac{k_{B} T}{\pi\left(E_{r}^{D}+E_{r}^{A}\right)}} \tanh \frac{e\left|V_{\text {bias }}\right|}{2 k_{B} T} \\
& \cdot \exp \left\{-\frac{\left[E_{r}^{A}-e V_{\text {bias }}+\left(e \xi_{A} \eta+e \gamma_{A} V_{\text {bias }}\right)\right]^{2}}{4 E_{r}^{A} k_{B} T}\right. \\
& \left.-\frac{\left[E_{r}^{D}-\left(e \xi_{D} \eta+e \gamma_{D} V_{\text {bias }}\right)\right]^{2}}{4 E_{r}^{D} k_{B} T}\right\} .
\end{aligned}
$$

b. Initial configuration $D^{+}-S-A$ : Both levels are initially vacant and above $\varepsilon_{\mathrm{FL}}$ and $\varepsilon_{\mathrm{FR}}$. Intramolecular ET is induced by donor level fluctuations to cross the Fermi level of the negatively biased electrode. Further relaxation of the occupied donor level brings this level to cross the vacant acceptor level, leading to intramolecular ET. The rate constant is

$$
\begin{aligned}
k_{D A}^{o / o}= & \kappa_{D A} \frac{\omega}{2 \pi} \sqrt{\frac{k_{B} T}{\pi E_{r}^{A}} \tanh \frac{e\left|V_{\text {bias }}\right|}{2 k_{B} T}} \\
& \cdot \exp \left\{-\frac{\left[E_{r}^{D}-\left(e \xi_{D} \eta+e \gamma_{D} V_{\text {bias }}\right)\right]^{2}}{4 E_{r}^{D} k_{B} T}\right. \\
& \left.-\frac{\left[E_{r}^{A}-\left(e \xi_{A} \eta+e \gamma_{A} V_{\text {bias }}\right)\right]^{2}}{4 E_{r}^{A} k_{B} T}\right\} .
\end{aligned}
$$

c. Initial configuration $D-S-A$ : This case is analogous to case (a). The rate constant is

$$
\begin{aligned}
k_{D A}^{r / r}= & \kappa_{D A} \frac{\omega}{2 \pi} \sqrt{\frac{k_{B} T}{\pi E_{r}^{D}}} \tanh \frac{e\left|V_{\text {bias }}\right|}{2 k_{B} T} \\
& \cdot \exp \left\{-\frac{\left[E_{r}^{A}-e V_{\text {bias }}+\left(e \xi_{A} \eta+e \gamma_{A} V_{\text {bias }}\right)\right]^{2}}{4 E_{r}^{A} k_{B} T}\right. \\
& \left.-\frac{\left[E_{r}^{D}-e V_{\text {bias }}+\left(e \xi_{D} \eta+e \gamma_{D} V_{\text {bias }}\right)\right]^{2}}{4 E_{r}^{D} k_{B} T}\right\} .
\end{aligned}
$$

\section{Rate constants at negative bias voltages}

ET is now from the negatively biased, right electrode to the positively biased, left electrode. The rate constants follow closely those for positive bias voltages. The results for the four electronic configurations in Fig. 5 are

$k_{A D}^{r / o}=k_{D A}^{r / o} \exp \left[\frac{\left(e \xi_{D} \eta+e \gamma_{D} V_{\text {bias }}\right)-\left(e \xi_{A} \eta+e \gamma_{A} V_{\text {bias }}\right)}{k_{B} T}\right]$,

$$
\begin{aligned}
& k_{A D}^{o / r}=k_{D A}^{o / r} \exp \left[-\frac{e V_{\text {bias }}+\left(e \xi_{D} \eta+e \gamma_{D} V_{\text {bias }}\right)-\left(e \xi_{A} \eta+e \gamma_{A} V_{\text {bias }}\right)}{k_{B} T}\right], \\
& k_{A D}^{r / r}=k_{D A}^{o / o} \exp \left[-\frac{\left(e \xi_{D} \eta+e \gamma_{D} V_{\text {bias }}\right)+\left(e \xi_{A} \eta+e \gamma_{A} V_{\text {bias }}\right)}{k_{B} T}\right], \\
& k_{A D}^{o / o}=k_{D A}^{r / r} \exp \left[-\frac{e V_{\text {bias }}-\left(e \xi_{D} \eta+e \gamma_{D} V_{\text {bias }}\right)+e V_{\text {bias }}-\left(e \xi_{A} \eta+e \gamma_{A} V_{\text {bias }}\right)}{k_{B} T}\right],
\end{aligned}
$$

where $k_{D A}^{r / o}, k_{D A}^{o / r}, k_{D A}^{o / o}$, and $k_{D A}^{r / r}$ are given by Eqs. (23)-(26).

\section{Total current for molecular rectification in the fully and partially adiabatic limits}

The total current at given overvoltage and given, positive or negative bias voltage, is

$$
\begin{aligned}
i^{\mathrm{coh}}= & e C\left[k_{D A}^{r / o} P_{D}\left(1-P_{A}\right) \theta\left(V_{\text {bias }}\right)-k_{A D}^{r / o} P_{A}\left(1-P_{D}\right)\right. \\
& \times \theta\left(-V_{\text {bias }}\right)+k_{D A}^{o / r} P_{A}\left(1-P_{D}\right) \theta\left(V_{\text {bias }}\right)-k_{A D}^{o / r} P_{D}(1 \\
& \left.-P_{A}\right) \theta\left(-V_{\text {bias }}\right)+k_{D A}^{o / o}\left(1-P_{D}\right)\left(1-P_{A}\right) \theta\left(V_{\text {bias }}\right) \\
& +k_{D A}^{r / r} P_{A} P_{D} \theta\left(V_{\text {bias }}\right)-k_{A D}^{o / o}\left(1-P_{A}\right)\left(1-P_{D}\right) \\
& \left.\times \theta\left(-V_{\text {bias }}\right)-k_{A D}^{r / r} P_{D} P_{A} \theta\left(-V_{\text {bias }}\right)\right] .
\end{aligned}
$$

$P_{D}$ and $P_{A}$ are again the donor and acceptor level populations, and $\theta(x)$ the step function. The superscript "coh" em- phasizes the coherent nature of these processes, in contrast to the sequential pattern in the fully diabatic limit. Equation (32) can be given the following other form:

$$
\begin{aligned}
i^{\mathrm{coh}}= & P_{D} P_{A}\left\{k _ { D A } ^ { r / o } \left[\frac{1-P_{A}}{P_{A}} \theta\left(V_{\text {bias }}\right)-\frac{k_{A D}^{r / o}}{k_{D A}^{r / o}} \frac{1-P_{D}}{P_{D}}\right.\right. \\
& \left.\times \theta\left(-V_{\text {bias }}\right)\right]+k_{D A}^{o / r}\left[\frac{1-P_{D}}{P_{D}} \theta\left(V_{\text {bias }}\right)-\frac{k_{A D}^{o / r}}{k_{D A}^{o / r}} \frac{1-P_{A}}{P_{A}}\right. \\
& \left.\times \theta\left(-V_{\text {bias }}\right)\right]+k_{D A}^{o / o}\left[\frac{1-P_{D}}{P_{D}} \frac{1-P_{A}}{P_{A}} \theta\left(V_{\text {bias }}\right)-\frac{k_{A D}^{r / r}}{k_{D A}^{o / o}}\right. \\
& \left.\times \theta\left(-V_{\text {bias }}\right)\right]+k_{D A}^{r / r}\left[\theta\left(V_{\text {bias }}\right)-\frac{k_{A D}^{o / o}}{k_{D A}^{r / r}} \frac{1-P_{D}}{P_{D}} \frac{1-P_{A}}{P_{A}}\right. \\
& \left.\left.\times \theta\left(-V_{\text {bias }}\right)\right]\right\},
\end{aligned}
$$


where the electrode coverage is taken to be unity. The level populations are

$$
\begin{aligned}
& P_{A}=\frac{1}{1+\exp \left(-\frac{e \xi_{A} \eta+e \gamma_{A} V_{\text {bias }}-e V_{\text {bias }}}{k_{B} T}\right)}, \\
& P_{D}=\frac{1}{1+\exp \left(-\frac{e \xi_{D} \eta+e \gamma_{D} V_{\text {bias }}}{k_{B} T}\right)}
\end{aligned}
$$

or

$$
\begin{aligned}
& \frac{1-P_{A}}{P_{A}}=\exp \left(-\frac{e \xi_{A} \eta+e \gamma_{A} V_{\text {bias }}-e V_{\text {bias }}}{k_{B} T}\right), \\
& \frac{1-P_{D}}{P_{D}}=\exp \left(-\frac{e \xi_{D} \eta+e \gamma_{D} V_{\text {bias }}}{k_{B} T}\right) .
\end{aligned}
$$

Together with the rate constants and currents in Sec. V, Eqs. (23) -(32) are the basis for the numerical analysis in the next section.

\section{NUMERICAL CURRENT-BIAS VOLTAGE RELATIONS IN MOLECULAR RECTIFICATION}

The analytical formalism in Secs. V and VI are illustrated in Figs. 7 and 8. The current-voltage relations shown are also representative of molecular donor-acceptor rectification in vacuum or gas ambient but here as noted, with no overvoltage. The equilibrium electronic energies are also different. They are represented in Eqs. (10)-(13) or (23)-(32) by the quantities $e \xi_{D} \eta$ and $e \xi_{A} \eta$ which assume formally the role of electronic energy levels at zero bias voltage.

\section{A. Totally diabatic limit}

The totally diabatic limit applies naturally only to the $D-S-A \rightarrow D^{+}-S-A^{-}$case. Reorganization free energies are taken in the range $0.1-0.5 \mathrm{eV}$. The oxidized and reduced level gaps are then $\approx 2 E_{r}^{D}$ for the donor and $\approx 2 E_{r}^{A}$ for the acceptor, or a donor-acceptor energy gap of $\approx E_{r}^{D}+E_{r}^{A}$ $\approx 0.2-1 \mathrm{eV}$ at equilibrium. Bias voltages are in range up to $\left|e V_{\text {bias }}\right| \leqslant E_{r}^{D}+E_{r}^{A}$. Overvoltages are confined within $\left|e \xi_{S} \eta\right| \leqslant E_{r}^{S}, S=D, A$.

Current-bias voltage relations for the totally diabatic limit are shown in Fig. 7. There is no rectification at small overvoltage where the occupied and vacant levels are symmetric with respect to the Fermi levels at zero bias voltage. Strong rectification, however, arises as the overvoltage increases (numerically) towards the reorganization free energies, or as level asymmetry in the gas phase are invoked. The currents are much higher for positive than for negative bias voltage, and increase with increasing $\eta$. There is also a threshold for strong current onset at $e V_{\text {bias }} \approx E_{r}^{D} / \xi_{D}$. The "rectification ratio," i.e., $i\left(+V_{\text {bias }}\right) / i\left(-V_{\text {bias }}\right)$ is $\approx 1.5$ when $\left|V_{\text {bias }}\right|=250 \mathrm{mV}$ and $\approx 2$ when $\left|V_{\text {bias }}\right|=350 \mathrm{mV}$, for $e \xi_{D} \eta$ $\approx 20 \mathrm{mV}$ and $e \xi_{A} \eta \approx 40 \mathrm{mV}$. These values increase to $\approx 3$ and $\approx 6$ when $e \xi_{D} \eta$ and $e \xi_{A} \eta$ increase to $35 \mathrm{mV}$ and $70 \mathrm{mV}$, respectively. The rectification ratio can take much higher values when the reorganization Gibbs free energy is larger (Fig. 7) or the interfacial transmission coefficients are significantly different. The former effect scales with the threshold voltage and allows a wider overpotential range, and therefore increased donor and acceptor level asymmetry.

\section{B. Totally and partially adiabatic limits}

These limits can be approached jointly and the currents scaled with respect to $\kappa_{D A}$ of the intramolecular ET step. They differ from the fully diabatic limit by the coherent feature and partial vibrational relaxation. Figure 8 shows current-bias voltage relations based on the full set of equations, Eqs. (32)-(35), and the rate constants, Eqs. (23)-(31). The four different transitions dominate individually in different overvoltage ranges which prepare the $D-S-A$ molecule in different equilibrium states. The different overvoltages correspond to different vacuum level positions at zero bias voltage. The correlations show:

(i) Pronounced current-voltage asymmetry, or rectification, is notable already at zero overvoltage. This reflects both the potential distribution in the gap and asymmetry in the mechanism where intramolecular and interfacial ET proceed coherently.

(ii) The asymmetry depends sensitively on the potential distribution $\left(\gamma_{D}\right.$ and $\left.\gamma_{A}\right)$, particularly at the donor site. When this variation is strong, i.e., $\gamma_{D}$ is large, current rectification is at negative bias voltage. Rectification is at positive bias voltage when the potential variation at the donor site is weak, or $\gamma_{D}$ small.

(iii) The rectification ratio reaches more than an order of magnitude already at fairly small bias voltages and is larger the higher the overpotential or level asymmetry, and the larger the reorganization Gibbs free energy.

\section{STRONG MOLECULE-ELECTRODE INTERACTION AND FANO EFFECTS}

We address briefly the case where either the donor or the acceptor level is broadened so strongly by the metallic continuua that the exchange interaction exceeds the nuclear reorganization free energy. ET between the electrode and the broadened level then no longer constitutes a separate transition, and the four-channel transition via separate donor and acceptor states reduces to a three-channel transition. The broadened level however, still depends on the nuclear configurations which affect the amount of charge on the adsorbate and the electronic level density, and therefore the intramolecular ET rate constants involving the broadened donor or acceptor level. Several patterns of rectification arise. Some of these resemble in situ STM of single-level redox centres and electrochemical ET via strongly adsorbed atoms or molecules.

\section{A. Broadened donor or acceptor level independent of environmental nuclear dynamics}

The rectification process here reduces to a three-channel transition. ET is either from the broadened donor level to the opposite electrode via the discrete acceptor level, or from a discrete donor level to the broadened acceptor level. This follows closely STM of redox molecules (Figs. 2 and 6). The STM formalism in previous reports ${ }^{30-32,56}$ therefore applies, 
except that the interfacial rate constants are replaced by rate constants for ET between the discrete and the broadened donor and acceptor levels. ${ }^{59,60}$ The diabatic rate constants $k_{\text {ox }}$ and $k_{\text {red }}$ in Eq. (35) of Ref. 34 are, for example, replaced by Eqs. (23) and (24) in Ref. 59.

\section{B. Configurational coordinate dependence of a broadened donor or acceptor level}

A self-consistent formalism incorporating the combined electronic and vibrational level broadening is available for electrochemical ET between a molecule in solution and an electronically broadened, solvent dependent adsorbate level. ${ }^{59}$ This does not presently carry over to STM of singlelevel redox adsorbates or molecular rectification. We provide instead current-bias voltage relations for a simpler case. The broadened level is specifically the donor level, $\varepsilon_{D}$ (Fig. 6) coupled to a set of environmental displaced harmonic nuclear coordinates, $\left\{q_{k}\right\}$ of vibrational frequencies $\left\{\omega_{k}\right\}$.

The density of electronic states, $\rho_{D L}$, caused by broadening of the donor level, $\varepsilon_{0 D}$, is

$$
\rho_{D L}=\frac{1}{\pi} \frac{\delta_{D}}{\left[\left(\varepsilon_{L}-\varepsilon_{\mathrm{FL}}\right)-\left(\varepsilon_{0 D}-\varepsilon_{\mathrm{FL}}\right)\right]^{2}+\delta_{D}^{2}},
$$

where $\delta_{D}$ is the donor level broadening caused by interaction with the adjacent electrode. Adding linear coupling terms along the $q_{k}$-modes, $\rho_{D L}$ becomes

$$
\begin{aligned}
\rho_{D L} & =\rho\left(\varepsilon_{L} ;\left\{q_{k}\left(\varepsilon_{L}\right)\right\}\right) \\
& =\frac{1}{\pi} \frac{\delta_{D}}{\left[\left(\varepsilon_{L}-\varepsilon_{\mathrm{FL}}\right)-\left(\varepsilon_{O D}-\varepsilon_{\mathrm{FL}}\right)-\sum_{k} g_{k}\left(q_{k}-q_{k 0 i}\right)\right]^{2}+\delta_{D}^{2}} .
\end{aligned}
$$

The meaning of the coupling constants, $\left\{g_{k}\right\}$, will be specified below. The rate constant, $k_{\mathrm{ox}}$, for ET from the electronically broadened donor level to the vacant acceptor level is

$$
\begin{aligned}
k_{\mathrm{ox}}= & \kappa_{D A} \frac{\omega_{\mathrm{eff}}}{2 \pi} \int d \varepsilon_{L} \rho_{D L}\left(\varepsilon_{L} ;\left\{q_{k}^{*}\left(\varepsilon_{L}\right)\right\}\right) f\left(\varepsilon_{L}\right) \\
& \times \exp \left[-\frac{U_{i}\left(\left\{q_{k}^{*}\right\}\right)-U_{i}\left(\left\{q_{k 0 i}\right\}\right)}{k_{B} T}\right],
\end{aligned}
$$

where $f\left(\varepsilon_{L}\right)$ is the Fermi function

$$
f\left(\varepsilon_{L}\right)=\left[1+\exp \left(\frac{\varepsilon_{L}-\varepsilon_{\mathrm{FL}}}{k_{B} T}\right)\right]^{-1},
$$

and $\left\{q_{k}^{*}\right\}$ are the values of $\left\{q_{k}\right\}$ at the saddle point of the crossing between the initial and final state potential surfaces (acceptor level vacant and occupied, respectively), determined by

$$
q_{k}^{*}=(1-\alpha) q_{k 0 i}+\alpha q_{k 0 f}=q_{k 0 i}+\alpha\left(q_{k 0 f}-q_{k 0 i}\right) .
$$

$q_{k 0 i}$ and $q_{k 0 f}$ are the equilibrium values of $q_{k}$ in the initial and final state where the acceptor level is vacant and occupied, respectively. The transfer coefficient, $\alpha$, is

$$
\alpha=\frac{1}{2}\left[1-\frac{e \xi_{A} \eta+e \gamma_{A} V_{\mathrm{bias}}+\left(\varepsilon_{L}-\varepsilon_{\mathrm{FL}}\right)}{E_{r}^{A}}\right] .
$$

With the assumption that the nuclear configuration is determined solely by ET at the acceptor site, the equilibrium coordinate displacement and reorganization free energy in Eqs. (40) and (41) are determined by the charge state of this site only.

By combining Eq. (38) with Eqs. (39)-(41) $k_{\text {ox }}$ is obtained in the immediately usable form,

$$
\begin{aligned}
k_{\mathrm{ox}}= & \kappa_{D A} \frac{\omega_{\mathrm{eff}}}{2 \pi} \int_{-\infty}^{\infty} d \varepsilon_{L} \frac{1}{\pi} \cdot \frac{\delta_{D}}{\left[\left(\varepsilon_{L}-\varepsilon_{\mathrm{FL}}\right)-\left(\varepsilon_{0 D}-\varepsilon_{\mathrm{FL}}\right)-\frac{1}{2}\left(1-\frac{e \xi_{A} \eta+e \gamma_{A} V_{\mathrm{bias}}+\left(\varepsilon_{L}-\varepsilon_{\mathrm{FL}}\right)}{E_{r}^{A}}\right) \sum_{k} g_{k}\left(q_{k 0 f}-q_{k 0 i}\right)\right]^{2}+\delta_{D}^{2}} \\
& \cdot \frac{1}{1+\exp \left(\frac{\varepsilon_{L}-\varepsilon_{\mathrm{FL}}}{k_{B} T}\right)} \exp \left\{-\frac{\left[E_{r}^{A}-e \xi_{A} \eta-e \gamma_{A} V_{\mathrm{bias}}-\left(\varepsilon_{L}-\varepsilon_{\mathrm{FL}}\right)\right]^{2}}{4 E_{r}^{A} k_{B} T}\right\} .
\end{aligned}
$$

The coupling constants $\left\{g_{k}\right\}$ are related to the reorganization free energy, $E_{r}^{A}$, by

$$
\frac{1}{2} \sum_{k} \frac{g_{k}^{2}}{\hbar \omega_{k}}=E_{r}^{A},
$$

and the observable current is related to $k_{\mathrm{ox}}$ by Eq. (24).

Further analysis of this limit and approaches to cases where both the donor and acceptor levels are electronically and vibrationally broadened will be provided elsewhere. ${ }^{60}$

\section{CONCLUDING REMARKS}

Sections V and VI offer an analytical theoretical frame for electron flow through an ordered assembly of donoracceptor molecules between two enclosing metallic electrodes. The formalism follows that for STM of redox molecules, ${ }^{32-36,58}$ but addresses one level higher complexity. Focus has been on current rectification as this device function in molecular electronics is coming within reach. ${ }^{11,12,16-22}$ Together with the theory of ex situ and in situ STM of redox molecules the formalism, however, holds 


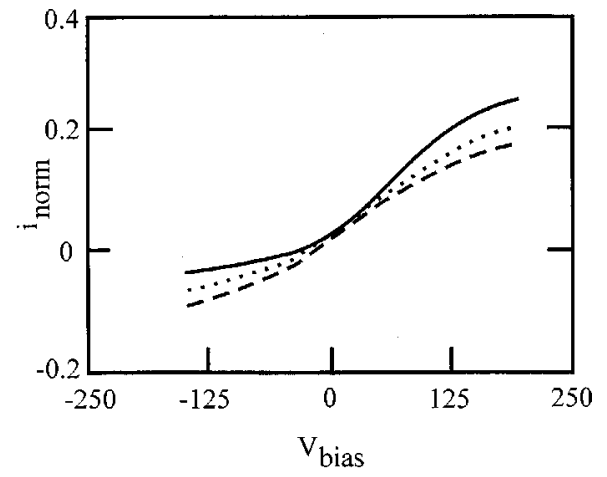

FIG. 7. Current/bias voltage relations for electron flow through the donoracceptor molecule. The current, $i_{\text {norm }}$, is scaled with the pre-exponential factor. $V_{\text {bias }}$ in $\mathrm{mV}$. Fully diabatic limit with equal interfacial transmission coefficients. Equilibrium level configuration as in Fig. 1. $E_{r}^{D}=E_{r}^{A}$ $=0.1 \mathrm{eV}, \gamma_{D}=0.2, \gamma_{A}=0.4$. Solid line: $e \eta \xi_{D}=35 \mathrm{mV}$, e $\eta \xi_{A}=70 \mathrm{mV}$. Dotted line: $e \eta \xi_{D}=10 \mathrm{mV}$, e $\eta \xi_{A}=20 \mathrm{mV}$. Dashed line: $e \eta \xi_{D}=20 \mathrm{mV}$, e $\eta \xi_{A}=40 \mathrm{mV}$.

promise as a broader frame for spectroscopic properties of single molecules and for more complex molecular assemblies and architectures. ${ }^{1-3,15}$

ET through two-level donor-acceptor molecules exhibits multifarious behavior. Two broad classes of mechanisms can be anticipated, with strong electronic-vibrational coupling and relations to interfacial ET in condensed media as common features. One class represents donor and acceptor levels that interact weakly with the enclosing metallic electrodes. Electronic conduction through the molecular layers is constituted by individual ET steps from the negatively to the positively biased electrode via the donor and acceptor levels. The sequence reduces to independent ET steps in the fully diabatic limit, where all the electronic interactions between the participating components (donor and acceptor fragments, the electrodes) is weak. This limit, however, strongly attenuates electron flow. Electronic conduction is much more facile when the electronic contacts between the molecular fragments and the electrodes are strong and the partially or fully adiabatic limit prevails. Both high interfacial ET rate constants and a different physical pattern then emerge, involving dynamically populated intermediate states. A conspicuous feature is that rectified ET proceeds through populated donor and acceptor intermediate states, with only partial vibrational relaxation. This follows patterns for adiabatic three-level STM processes. ${ }^{34,36}$ The additional intramolecular ET step in the donor-acceptor rectifier means that two electrons, instead of several, are transferred in a single reactive event. Focus should thus be on the partially and fully adiabatic limits in approaches to a real molecular rectifying device.

The other class of mechanisms involves so strong interactions with the metallic surfaces, that electronic broadening compares with vibrational broadening. The metal and adsorbed molecular fragment then constitute a single element, and rectification reduces to a three-level transition. The density of states of the broadened level is determined by the combined adsorbate-metal and vibrational broadening. Such mechanisms resemble three-level ET in STM (Refs. 33-36) and electrochemical ET from a molecule in solution ${ }^{59}$ to an electronically broadened adsorbate level.
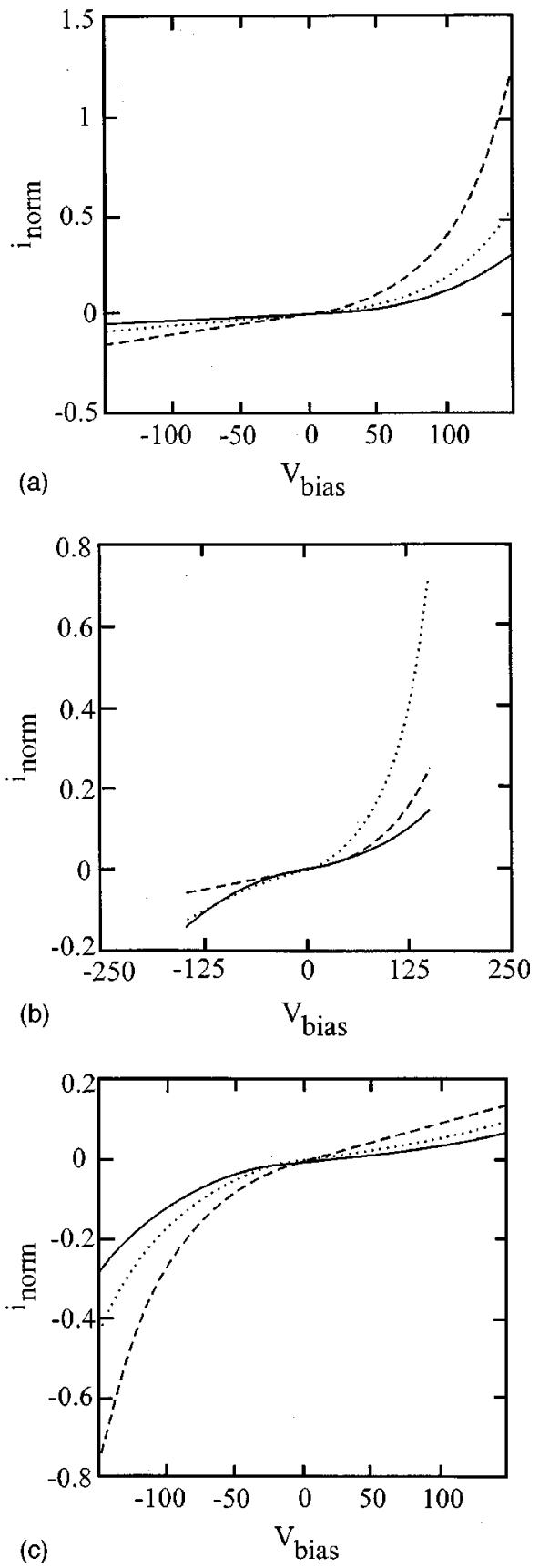

FIG. 8. Current/bias voltage relations in the partially and fully adiabatic limits. Scales as in Fig. 7. Reorganization free energies $E_{r}^{D}=E_{r}^{A}=0.2 \mathrm{eV}$. Different potential distributions in the gap. (a) $\gamma_{D}=0.2, \gamma_{A}=0.4$. Solid line: $e \eta \xi_{D}=e \eta \xi_{A}=0$. Dotted line: $e \eta \xi_{D}=-10 \mathrm{mV}$, e $\eta \xi_{A}=-20 \mathrm{mV}$. Dashed line: $e \eta \xi_{D}=-25 \mathrm{mV}$, e $\eta \xi_{A}=-50 \mathrm{mV}$. (b) Solid line: $\gamma_{D}=0.25, \gamma_{A}$ $=0.75 .: \quad e \eta \xi_{D}=e \eta \xi_{A}=0 . \quad$ Dotted line: $\gamma_{D}=0.1, \quad \gamma_{A}=0.5$. e $\eta \xi_{D}$ $=-7.5 \mathrm{mV}, \quad e \eta \xi_{A}=-38 \mathrm{mV}$. Dashed line: $\gamma_{D}=0.1, \gamma_{A}=0.5$, e $\eta \xi_{D}$ $=2.5 \mathrm{mV}$, e $\eta \xi_{A}=12.5 \mathrm{mV}$. (c) $\gamma_{D}=0.45, \gamma_{A}=0.9$. Solid line: $e \eta \xi_{D}$ $=e \eta \xi_{A}=0$. Dotted line: $e \eta \xi_{D}=-10 \mathrm{mV}$, e $\eta \xi_{A}=-20 \mathrm{mV}$. Dashed line: e $\eta \xi_{D}=-25 \mathrm{mV}$, e $\eta \xi_{A}=-38 \mathrm{mV}$.

The first class of mechanisms is addressed in Sec. $\mathrm{V}$ for the fully diabatic, and in Sec. VI for the partially and fully adiabatic limits. Both are characterized by their steady-state currents determined by the individual interfacial and intramolecular ET rate constants and the populations of the donor and acceptor fragments in different electronic states. The rate constants are determined by the electronic energies 
of the donor and acceptor fragments, the nuclear reorganization free energies, the overpotential and the bias potential, the electronic transmission coefficients, and the potential distribution in the gap between the electrodes. The electron flow in the partially and fully adiabatic limits is, moreover, controlled by several reactive channels working in parallel. The activation free energies of the rate constants resemble those for in situ STM (Refs. 32-36, 58) and interfacial electrochemical ET (Refs. 53, 54) but the electronic and vibrational level widths are different and the voltage ranges of the different channels crucial. The rectified current pattern is therefore also different from the overvoltage and bias potential dependent currents in electrochemical ET and in situ STM.

The limit of strong electronic contact between the $D-S-A$ molecule and one of the metallic surfaces, and the resulting Fano broadening is addressed in Sec. VIII. The most important result is Eq. (42), which points to a clear relation to in situ STM of redox molecules. ${ }^{34,36,58}$ The broadened level and contacting metal thus constitute now a single channel, and the rectification process is reduced to a threelevel process. Current/voltage relations therefore follow "spectroscopic" patterns similar to those for in situ STM.

The formalism accounts for threshold bias voltage effects and strong current asymmetry in the donor-acceptor configurations in Figs. 1 and 3-5, with much higher currents generally for positive than for negative bias voltage (see, however, the section below). The patterns are determined by the donor and acceptor energies relative to the Fermi energies of the electrodes, the electronic-vibrational coupling (reorganization energies), the overvoltage, the potential distribution in the gap, and the diabatic or adiabatic nature of the process. Current rise sets in when the donor level is brought to cross the Fermi level of the negatively biased electrode. The threshold voltage is therefore higher the larger the reorganization energy and the lower the overvoltage. "Rectification ratios," i.e., $i\left(+V_{\text {bias }}\right) / i\left(-V_{\text {bias }}\right)$ are in the range 1.5-6 for appropriate parameter values, $E_{r}^{S}<0.5 \mathrm{eV}$, $S=D, A,\left|V_{\text {bias }}\right|<0.5 \mathrm{~V},|\eta|<0.2 \mathrm{~V}$ in the totally diabatic limit. The ratio is higher for larger energy gaps and $E_{r}^{S}$. High "rectification ratios" are, however, better achieved in the partially or fully adiabatic limits, where the "rectification ratio" reaches more than an order of magnitude already for modest reorganization energies and overvoltage [Figs. 8(a) and $8(\mathrm{~b})$ ]. The ratio also depends sensitively on the bias voltage distribution in the gap [Fig. 8(c)]. Current onset can be at positive or negative bias voltage when the potential variation at the donor site is weak and strong, respectively, although the former would be the more appropriate for actual rectifying molecules.

Focus in the approach in Secs. V-VIII was on nuclear motion, strong electronic-vibrational coupling, and vibrationally induced resonance with the molecular redox levels. In these respects the approach differs from most recent reports on electronic conduction through molecular wires, where attention was mostly on the electronic factor. Analysis of environmental effects on the electronic features in some reports, however, has some bearing on the results above. Ratner and associates addressed the effects of electronic diagonal and off-diagonal disorder. ${ }^{24,25,31}$ These notions refer to the energy and transfer integrals, respectively. In the superexchange mode the observations would relate to the transmission coefficients in the fully diabatic and partially adiabatic limits of the formalism in Secs. V and VI. Disorder decreases the conductivity close to electronic resonance and induces electron localization, but enhances the conductivity when the molecular levels are off resonance with the metallic Fermi levels. The latter effect was also observed in an early report. $^{61}$

The mechanism of the molecular resonant tunnel diode of Reed and associates ${ }^{18}$ proposed by Seminario et al. ${ }^{27,28}$ illustrates other features of importance in relation to nuclear dynamic effects. The current is mediated by the LUMO, the character of which changes drastically with the molecular charge. When the molecule goes from neutral to being single negatively charged the LUMO changes from being partly localized to being delocalized over the entire molecule, opening an efficient ET channel. Double charging again leads to orbital localization and closing of the channel. These effects are accompanied by increasing molecular structural planarity as the negative charge increases, indicative that conductivity is controlled both by charging and vibrational motion. Such effects should be incorporated in the formalism of Secs. V and VI, by introducing additional gating modes such as known in other contexts. ${ }^{53,54}$

Organized monolayers of existing molecular wire types with accessible electronic states suitable for temporary electronic population are appropriate target molecules for the molecular diode formalism in Secs. V and VI, and the recent theory of STM. ${ }^{32-36,58}$ Some systems were noted in Sec. II. Other cases for facile asymmetric current/bias voltage relations and electron flow along molecular wires, could include: (a) self-assembled condensed porphyrin complexes; ${ }^{62,63}$ (b) condensed aromatic ring systems, ${ }^{9,64}$ and multiply functionalized thiolates; ${ }^{20}$ (c) carbon nanotube systems; $8,65,66$ (d) supramolecular ladders and other arrays of large transition metal complexes; ${ }^{67,68}$ (e) single molecules in metallic nanogaps; ${ }^{69,70}$ (f) differential resistance patterns in adsorbed heteropolytungstate complexes $;^{71}$ (g) molecular photodiodes; ${ }^{13,14,72}$ and (h) perhaps axial conduction along doublestranded oligonucleotides. ${ }^{73}$ We conclude by addressing briefly recent data for current rectification in monolayers of hexadecyl-quinolinium tricyanodimethanide, ${ }^{11,12,30}$ the following features of which are important in relation to the formalism and computations above:

(i) Mono- and multilayer films were characterized comprehensively. New synthetic methods were used, and samples characterized by spectroscopic methods, electrochemistry, x-ray scattering, LangmuirBlodgett techniques, and theoretical methods.

(ii) The molecule holds a quaternary quinolinium unit tied to a hexadecyl $(T)$ chain, HDQ, and a 3-cyanoquinodimethanide unit, $3 \mathrm{CNQ}$. The ground state is, schematically $T-D^{+}-S-A^{-} \leftrightarrow T-D-S-A, D^{+}$denotes the HDQ, $A$ the 3 CNQ units, and $S$ the aromatic spacer between $D$ and $A$. 
(iii) The molecules are inserted between electrodes of gold, aluminum or graphite, and deposited aluminum or magnesium layers. Electron flow on metallic substrates is from the hydrophilic 3CNQ end to the hydrophobic HDQ end, corresponding to Fig. 1.

(iv) The ground state is a resonance between a zwitterion and neutral form, cf. above. ${ }^{74}$ There is a low-lying charge transfer state of charge separated biradical nature corresponding to a strong absorption band at $15.300 \mathrm{~cm}^{-1}$ or $1.9 \mathrm{eV}^{75}$ The transition is at a higher energy, i.e., $17.700 \mathrm{~cm}^{-1}$ or $2.2 \mathrm{eV}$ in LangmuirBlodgett films of the molecule. ${ }^{75}$

(v) The spectral bandwidth of the charge transfer transition is about $3.700 \mathrm{~cm}^{-1}$ or $0.46 \mathrm{eV}$ in the LangmuirBlodgett film, and $0.60 \mathrm{eV}$ for the molecule in acetonitrile solution. ${ }^{75}$ These values correspond to significant nuclear reorganization energies, i.e., 0.53 and $0.90 \mathrm{eV}$, respectively.

(vi) The current-voltage relation displays strong rectification with much higher currents at positive bias voltages when the molecule is oriented with the 3CNQ end towards the negatively biased electrode. There is a threshold voltage at $V_{\text {bias }} \approx+1.5 \mathrm{~V}$. Rectification ratios range from 2.4 to 26 . The threshold voltage depends insignificantly on the temperature but the transition from "blocked" to rectified current is sharper at $105 \mathrm{~K}$ and the absolute current values at given bias voltage much smaller than at room temperature.

(vii) Figure 1 accords qualitatively with the data, i.e., the ground state HOMO of the molecule is below and the excited state LUMO above the electrode Fermi levels at zero bias voltage. If the first intermediate state, $d_{1}$, Fig. 3, is roughly represented by the optical charge transfer state, then the observed threshold voltage accords roughly with the excitation energy of the charge transfer state, corresponding to parallel shift of the charge transfer state energy with the bias voltage. This could be the case but is far from certain as the potential distribution over the molecule in the gap is not known.

The data hold two other suggestions relating to Secs. V and VI. One is that the measured currents are very small. ${ }^{11,12}$ This could either be because only a small fraction of the molecules are active, or because the measured currents begin when there is still a significant energy gap between the donor and acceptor states. The latter would be in keeping with the substantial temperature coefficient (activation energy) of the current. The other suggestion is that the reorganization energy determined from the optical charge transfer band width is significantly smaller than the threshold voltage. This could imply that the intermediate state where the acceptor level is in the reduced state remains above the Fermi level of the positively biased electrode. The rectification process would then, interestingly, involve this state in a dynamically populated mode.

\section{ACKNOWLEDGMENTS}

Financial support from the Danish Technical Science Research Council, the EU program INTAS (Grant No. 991093), and Russian Foundation for Basic Research (Grant No. 00-03-32239a) is acknowledged.

\section{APPENDIX A: DERIVATION OF THE RATE CONSTANT, EQ. (23)}

The intramolecular ET rate constant is, in the classical $\operatorname{limit}^{53}$

$$
\begin{aligned}
k_{D A}= & Q_{\dot{q}}^{-1} \int d \dot{q}_{n} \dot{q}_{n} P_{\mathrm{LZ}} \exp \left(-\frac{E_{\mathrm{kin}}}{k_{B} T}\right) Q_{q}^{-1} \int_{S^{*}} d q_{D} d q_{A} \\
& \times \exp \left[-\frac{U_{i}\left(q_{D}, q_{A}\right)}{k_{B} T}\right] .
\end{aligned}
$$

$E_{\text {kin }}$ is the kinetic energy and $\dot{q}_{n}$ is the velocity component normal to the crossing surface between the initial and final state potential surfaces, $S^{*} . U_{i}\left(q_{D}, q_{A}\right)$ is the potential energy in the initial state, $Q_{\dot{q}}$ the normalization of the (Maxwell) velocity distribution and $Q_{q}$ the normalization of the (Boltzmann) coordinate distribution.

The adiabatic Landau-Zener transition probability is $P_{\mathrm{LZ}}=1$. The first integral then gives

$$
\begin{aligned}
& Q_{\dot{q}}^{-1} \int d \dot{q}_{n} \dot{q}_{n} \exp \left(-\frac{E_{\mathrm{kin}}}{k_{B} T}\right)=\omega \sqrt{\frac{k_{B} T}{2 \pi \hbar \omega}}, \\
& \text { since } E_{\mathrm{kin}}=\frac{1}{2} \frac{\hbar}{\omega}\left(\dot{q}_{D}^{2}+\dot{q}_{A}^{2}\right) .
\end{aligned}
$$

Integration over the donor and acceptor coordinates, $q_{D}$ and $q_{A}$ in the second integral is conveniently replaced by integration over the donor and acceptor level energies. By definition,

$$
\begin{array}{rl}
\int_{S^{*}} & d q_{D} d q_{A} \exp \left[-\frac{U_{i}\left(q_{D}, q_{A}\right)}{k_{B} T}\right] \\
= & \int d q_{D} d q_{A}\left|\nabla\left(U_{i}-U_{f}\right)\right| \exp \left[-\frac{U_{i}\left(q_{D}, q_{A}\right)}{k_{B} T}\right] \\
& \times \delta\left(U_{i}-U_{f}\right),
\end{array}
$$

where $\boldsymbol{\nabla}\left(U_{i}-U_{f}\right)$ is the gradient of the potential surface difference at the crossing between the two surfaces. From Eqs. (15) and (16) the integral in Eq. (A3) can be recast as

$$
\begin{aligned}
\int d q_{D} d q_{A} \cdots & \rightarrow \frac{1}{g_{D}} \frac{1}{g_{A}} \int d \varepsilon_{D} d \varepsilon_{A} \cdots \delta\left(\varepsilon_{A}-\varepsilon_{D}\right) \\
& =\frac{1}{g_{D}} \frac{1}{g_{A}} \int d \varepsilon_{D} \exp \left[-\frac{U_{i}\left(\varepsilon_{A}=\varepsilon_{D}, \varepsilon_{D}\right)}{k_{B} T}\right],
\end{aligned}
$$

where $g_{D}$ and $g_{A}$ are the electronic-vibrational coupling constants, cf. Eq. (17),

$$
g_{D}=\sqrt{2 E_{r}^{D} \hbar \omega} ; \quad g_{A}=\sqrt{2 E_{r}^{A} \hbar \omega} .
$$

Expressing $q_{D}$ and $q_{A}$ in $U_{i}\left(q_{D}, q_{A}\right)$ in terms of $\varepsilon_{D}$ and $\varepsilon_{A}$ we obtain for the rate constant, 


$$
k_{D A}=\frac{\omega}{2 \pi} \Delta^{-1} \int_{\varepsilon_{\mathrm{FR}}}^{\varepsilon_{\mathrm{FL}}} d \varepsilon_{D} \exp \left[-\frac{U_{i}\left(\varepsilon_{A}=\varepsilon_{D}, \varepsilon_{D}\right)}{k_{B} T}\right],
$$

where the quantity, $\Delta$, is

$$
\Delta=\sqrt{\frac{4 \pi E_{r}^{D} E_{r}^{A} k_{B} T}{E_{r}^{D}+E_{r}^{A}} .}
$$

Since

$$
\left|\nabla\left(U_{i}-U_{f}\right)\right|=\sqrt{g_{D}^{2}+g_{A}^{2}}=\sqrt{2\left(E_{r}^{D}+E_{r}^{A}\right) \hbar \omega}
$$

and cf. Eqs. (15)-(17),

$$
\begin{aligned}
& \varepsilon_{S}=\varepsilon_{D}^{\mathrm{ox}}-g_{S} q_{S} ; \quad \text { or } q_{S}=\frac{1}{g_{S}}\left(\varepsilon_{S}-\varepsilon_{D}^{\mathrm{ox}}\right) ; \quad S=D, A, \\
& U_{i}\left(\varepsilon_{A}, \varepsilon_{D}\right)=\frac{1}{4 E_{r}^{A}}\left(\varepsilon_{A}-\varepsilon_{A}^{\mathrm{ox}}\right)^{2}+\frac{1}{4 E_{r}^{D}}\left(\varepsilon_{D}-\varepsilon_{D}^{\mathrm{ox}}-2 E_{r}^{D}\right)^{2},
\end{aligned}
$$

then $\Delta$ is noted to be identical to the width of the Gaussian integrand in Eq. (A6). Integration with respect to $\varepsilon_{D}$ gives the rate constant,

$$
k_{D A}=\frac{\omega}{2 \pi} \Delta^{-1} \Delta \varepsilon \exp \left[-\frac{U_{i}\left(\varepsilon_{A}^{*}=\varepsilon_{D}^{*}, \varepsilon_{D}^{*}\right)}{k_{B} T}\right],
$$

where the activation (free) energy takes the form in Eqs. (23) and (A9). The energy width, $\Delta \varepsilon$, is controlled by the thermal donor and acceptor level broadening if this broadening, given by Eq. (A7), is smaller than the bias voltage, $e V_{\text {bias }}$. If the thermal level broadening is larger than $e V_{\text {bias }}$, then $\Delta \varepsilon$ coincides approximately with the latter, i.e.,

$$
\begin{aligned}
& \Delta \varepsilon \approx \Delta \quad \text { if } \Delta<e V_{\text {bias }}, \\
& \Delta \varepsilon \approx e V_{\text {bias }} \text { if } \Delta>e V_{\text {bias }} .
\end{aligned}
$$

The following interpolation formula bridges these two limits:

$$
\Delta \varepsilon=\Delta \tanh \frac{e V_{\text {bias }}}{\Delta} .
$$

Equations (A7) and (A12) give the rate constant in Eqs. (23) and (24). The transmission coefficient, $\kappa_{D A}$, has been introduced to include the diabatic limit of intramolecular ET.

\section{APPENDIX B: DERIVATION OF EQS. (25)-(27) FOR THE $D^{+}-S-A^{-}, D^{+}-S-A^{+}$, AND $D-S-A^{-}$ CONFIGURATIONS}

In the initial state equilibrium configuration, $D^{+}-S-A^{-}, \varepsilon_{D}^{\mathrm{ox}}$ is located above $\varepsilon_{\mathrm{FL}}$ and $\varepsilon_{A}^{\text {red }}$ below $\varepsilon_{\mathrm{FR}}$. Configurational fluctuations bring $\varepsilon_{D}^{\text {ox }}$ to cross $\varepsilon_{\mathrm{FL}}$ and $\varepsilon_{A}^{\text {red }}$ to $\operatorname{cross} \varepsilon_{\mathrm{FR}} \cdot \varepsilon_{D}^{\mathrm{ox}}$ then becomes occupied by fast adiabatic ET from the negatively biased electrode to the left, while $\varepsilon_{A}^{\text {red }}$ becomes vacant by fast ET to the positively biased electrode to the right. Subsequent to interfacial ET the occupied donor level relaxes downhill and the vacant acceptor level uphill. They cross during vibrational relaxation, leading to intramolecular ET from the temporarily filled donor level to the temporarily vacant acceptor level. The nuclear velocities along the coordinates $q_{D}$ and $q_{A}$, strictly speaking, differ from the thermal value at the moment of intramolecular ET but this effect is small and disregarded. Focus is instead on the probability that the two original levels are brought to cross the appropriate Fermi levels, at the surface $S^{*}$ which separates the initial and final state potential surfaces in the $q_{D}, q_{A}$-plane.

This surface is shown in Fig. 9. $q_{A 0}$ is the coordinate displacement along $q_{A}$ when the acceptor level is occupied. The equilibrium values of $q_{D}$ and $q_{A}$ in the vacant states are at $q_{D}=q_{A}=0$, Eqs. (15)-(17). $\phi\left(q_{A}, q_{D L}\right)$ is the angle between the $q_{D}$-axis and trajectories crossing $q_{D L}$ of the separating surface. $\theta\left(q_{D}, q_{A R}\right)$ is the angle between the $q_{A}$-axis and trajectories crossing $q_{A R}$ of the separating surface. Following the procedure in Appendix A we can write the rate constant as

$$
k_{D A}=\omega \sqrt{\frac{k_{B} T}{2 \pi \hbar \omega}} \frac{\hbar \omega}{2 \pi k_{B} T} \Phi .
$$

The factors $\omega \sqrt{k_{B} T / 2 \pi \hbar \omega}$ and $\hbar \omega / 2 \pi k_{B} T$ derive from the kinetic energy and the normalizing factor, $Q_{q}^{-1}$, respectively. The function $\Phi$ is

$$
\begin{aligned}
\Phi= & \int_{q_{A R}}^{q_{A L}} d q_{A} \cos \varphi\left(q_{A}, q_{D L}\right) \\
& \times \exp \left[-\frac{\frac{1}{2} \hbar \omega\left(q_{A}-q_{A 0}\right)+\frac{1}{2} \hbar \omega q_{D L}^{2}}{k_{B} T}\right] \\
& +\int_{q_{D L}}^{q_{D R}} d q_{D} \cos \theta\left(q_{D}, q_{A R}\right) \\
& \times \exp \left[-\frac{\frac{1}{2} \hbar \omega\left(q_{A R}-q_{A 0}\right)+\frac{1}{2} \hbar \omega q_{D}^{2}}{k_{B} T}\right] .
\end{aligned}
$$

The angles $\varphi\left(q_{A}, q_{D L}\right)$ and $\theta\left(q_{D}, q_{A R}\right)$ are

$$
\cos \varphi=\frac{q_{D L}}{\sqrt{q_{D L}^{2}+\left(q_{A}-q_{A 0}\right)^{2}}} ; \quad \cos \theta=\frac{q_{A R}}{\sqrt{q_{D}^{2}+q_{A R}^{2}}} .
$$

Insertion of Eq. (B3) into Eq. (B2) and integration gives approximately

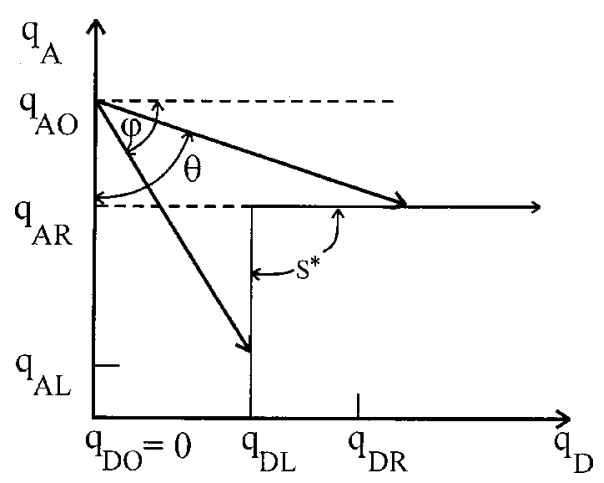

FIG. 9. Trajectories and crossing surface in the $q_{D}, q_{A}$-plane. The equilibrium values of $q_{D}$ and $q_{A}$, and the values where they cross the Fermi levels of the left and right electrodes, at positive bias of the electrode to the right are also shown. 


$$
\begin{aligned}
\Phi \approx & \frac{q_{D L}}{\sqrt{q_{D L}^{2}+\left(q_{A R}-q_{A 0}\right)^{2}}} \Delta_{q_{A}} \\
& \times \exp \left[-\frac{\frac{1}{2} \hbar \omega\left(q_{A R}-q_{A 0}\right)^{2}+\frac{1}{2} \hbar \omega q_{D L}^{2}}{k_{B} T}\right] \\
& +\frac{q_{A R}}{\sqrt{q_{A R}^{2}+q_{D L}^{2}}} \Delta_{q_{D}} \exp \left[-\frac{\frac{1}{2} \hbar \omega\left(q_{A R}-q_{A 0}\right)^{2}+\frac{1}{2} \hbar \omega q_{D L}^{2}}{k_{B} T}\right],
\end{aligned}
$$

where the widths are

$$
\begin{aligned}
& \frac{1}{\Delta_{q_{A}}}=\frac{\hbar \omega}{k_{B} T}\left(q_{A 0}-q_{A R}\right) \\
& =\frac{\hbar \omega}{k_{B} T}\left(\sqrt{\frac{2 E_{r}^{A}}{\hbar \omega}}-\frac{E_{r}^{A}+e V_{\mathrm{bias}}-\left(e \xi_{A} \eta+e \gamma_{A} V_{\mathrm{bias}}\right)}{\sqrt{2 E_{r}^{A} \hbar \omega}}\right) \\
& =\frac{\hbar \omega}{k_{B} T} \frac{1}{\sqrt{2 E_{r}^{A} \hbar \omega}}\left[E_{r}^{A}-e V_{\mathrm{bias}}+\left(e \xi_{A} \eta+e \gamma_{A} V_{\mathrm{bias}}\right)\right] \\
& \approx \frac{\hbar \omega}{k_{B} T} \sqrt{\frac{E_{r}^{A}}{2 \hbar \omega}} \\
& \frac{1}{\Delta_{q_{D}}}=\frac{\hbar \omega}{k_{B} T} \frac{1}{\sqrt{2 E_{r}^{D} \hbar \omega}}\left[E_{r}^{D}-\left(e \xi_{D} \eta+e \gamma_{D} V_{\mathrm{bias}}\right)\right] \\
& \approx \frac{\hbar \omega}{k_{B} T} \sqrt{\frac{E_{r}^{D}}{2 \hbar \omega}}
\end{aligned}
$$

Inserting $q_{D L}$ and $q_{A R}$ from Eq. (21) gives Eq. (25) where the transmission coefficient $\kappa_{D A}$ and the interpolation formula, Eq. (A11) have been added. Derivation of Eqs. (26) and (27) follows similar patterns.

\footnotetext{
${ }^{1}$ Molecular Electronic Devices, edited by F. L. Carter (Marcel Dekker, New York, 1982).

${ }^{2}$ Molecular Electronic Devices, edited by F. L. Carter, R. E. Siatkowski, and H. Wohltgen (Marcel Dekker, New York, 1988).

${ }^{3}$ Molecular Electronics: Science and Technology, edited by A. Aviram and M. Ratner (Annals of New York Academy of Sciences, New York, 1998), Vol. 852.

${ }^{4}$ A. Aviram and M. A. Ratner, Chem. Phys. Lett. 29, 277 (1974).

${ }^{5}$ N. J. Geddes, J. R. Sambles, D. J. Jarvis, W. G. Parker, and D. J. Sandman, Appl. Phys. Lett. 56, 1916 (1990)

${ }^{6}$ A. S. Martin, J. R. Sambles, and G. J. Ashwell, Phys. Rev. Lett. 70, 218 (1993).

${ }^{7}$ A. Dhirani, P.-H. Lin, P. Guyot-Sionnest, R. W. Zehner, and L. R. Zita, J. Chem. Phys. 106, 5249 (1997).

${ }^{8}$ P. G. Collins, A. Zettl, H. Brando, A. Thess, and R. E. Smalley, Science 278, 100 (1997).

${ }^{9}$ A. Stabel, P. Herwig, K. Müllen, and J. P. Rabe, Angew. Chem. 107, 1768 (1995).

${ }^{10}$ M. Pomerantz, A. Aviram, R. A. McCorkle, L. Li, and A. G. Schrott, Science 255, 1115 (1992).

${ }^{11}$ R. M. Metzger, B. Chen, U. Höpfner et al., J. Am. Chem. Soc. 119, 10455 (1997).

${ }^{12}$ R. M. Metzger, Acc. Chem. Res. 32, 950 (1999).

${ }^{13}$ M. Fujihira, in Nanostructures Based on Molecular Materials, edited by W. Göpel and C. Ziegler (Verlag Chemie, Weinheim, 1992).

${ }^{14}$ M. Fujihira, Ann. N.Y. Acad. Sci. 852, 306 (1998).

${ }^{15}$ C. P. Collier, E. W. Wong, M. Belohradsky, F. M. Raymo, J. F. Stoddart, P.
}

J. Kuekes, R. S. Williams, and J. R. Heath, Science 285, 391 (1999).

${ }^{16}$ M. A. Reed, C. Zhou, C. J. Muller, T. P. Burgin, and J. M. Tour, Science 278, 252 (1997).

${ }^{17}$ C. Zhou, M. R. Deshpande, M. A. Reed, L. Jones II, and J. M. Tour, Appl. Phys. Lett. 71, 611 (1997).

${ }^{18}$ J. Chen, M. A. Reed, A. M. Rawlett, and J. M. Tour, Science 286, 1550 (1999).

${ }^{19}$ J. Chen, W. Wang, M. A. Reed, A. M. Rawlett, D. W. Price, and J. M. Tour, Appl. Phys. Lett. 77, 1224 (2000).

${ }^{20}$ M. P. Samanta, W. Tian, S. Datta, J. I. Henderson, and C. P. Kubiak, Phys. Rev. B 53, R7626 (1996).

${ }^{21}$ S. Datta, W. Tian, S. Hong, R. Reifenberger, J. I. Henderson, and C. P. Kubiak, Phys. Rev. Lett. 79, 2530 (1997).

${ }^{22}$ S. Datta, Electronic Transport in Mesoscopic Systems (Cambridge University Press, Cambridge, 1997).

${ }^{23}$ V. Mujica, M. Kemp, and M. A. Ratner, J. Chem. Phys. 101, 6849 (1994); 101, 6856 (1994).

${ }^{24}$ V. Mujica, M. Kemp, and M. A. Ratner, J. Chem. Phys. 101, 5172 (1994).

${ }^{25}$ M. Kemp, A. Roitberg, V. Mujica, T. Wanta, and M. A. Ratner, J. Phys. Chem. 100, 8349 (1996).

${ }^{26}$ W. Tian, S. Datta, S. Hong, R. Reifenberger, J. I. Henderson, and C. P. Kubiak, J. Chem. Phys. 109, 2874 (1998).

${ }^{27}$ J. M. Seminario, A. G. Zacarias, and J. M. Tour, J. Phys. Chem. A 103, 7883 (1999).

${ }^{28}$ J. M. Seminario, A. G. Zacarias, and J. M. Tour, J. Am. Chem. Soc. 122, 3015 (2000).

${ }^{29}$ L. E. Hall, J. R. Reimers, N. S. Hush, and K. Silverbrook, J. Chem. Phys. 112, 1510 (2000).

${ }^{30}$ C. Krzeminski, C. Delerue, G. Allan, D. Vuillaumè, and R. M. Metzger, Phys. Rev. B 64, 085405 (2001).

${ }^{31}$ M. Olson, Y. Mao, T. Windus, M. Kemp, M. A. Ratner, N. Léon, and V. Mujica, J. Phys. Chem. B 102, 941 (1998).

${ }^{32}$ A. M. Kuznetsov, P. Sommer-Larsen, and J. Ulstrup, Surf. Sci. 275, 52 (1992).

${ }^{33}$ E. P. Friis, Yu. I. Kharkats, A. M. Kuznetsov, and J. Ulstrup, J. Phys. Chem. A 102, 7851 (1998).

${ }^{34}$ A. M. Kuznetsov and J. Ulstrup, J. Phys. Chem. A 104, 11531 (2000).

${ }^{35}$ H. Sumi, J. Phys. Chem. B 102, 1833 (1998).

${ }^{36}$ J. Zhang, Q. Chi, A. M. Kuznetsov, A. G. Hansen, H. Wackerbarth, H. E. M. Christensen, J. E. T. Andersen, and J. Ulstrup, J. Phys. Chem. (in press).

${ }^{37}$ N. Tao, Phys. Rev. Lett. 76, 4066 (1996).

${ }^{38}$ N. Tao, C. Z. Li, and H. X. He, J. Electroanal. Chem. 492, 81 (2000).

${ }^{39}$ W. Han, E. N. Durantini, T. A. Moore, A. L. Moore, D. Gust, P. Rex. G. Leatherman, G. R. Deely, N. Tao, and S. M. Lindsay, J. Phys. Chem. B 101, 10719 (1997).

${ }^{40}$ S. Yoshimoto, N. Hirakawa, K. Nishiyama, and I. Taniguchi, Langmuir 16, 4399 (2000)

${ }^{41}$ S. R. Snyder and H. S. White, J. Electroanal. Chem. 394, 177 (1995).

${ }^{42}$ X. D. Cui, A. Primak, X. Zarate, J. Tomfohr, O. F. Sankey, A. L. Moore, T. A. Moore, D. Gust, G. Harris, and S. M. Lindsay, Science 294, 571 (2001)

${ }^{43}$ A. M. Kuznetsov and J. Ulstrup, Chem. Phys. 157, 25 (1991).

${ }^{44}$ G. Iversen, Yu. I. Kharkats, A. M. Kuznetsov, and J. Ulstrup, Adv. Chem. Phys. 106, 453 (1999).

${ }^{45}$ X. Lu and K. W. Hipps, J. Phys. Chem. 101, 5391 (1991).

${ }^{46}$ L. Scudiero, D. E. Barlow, and K. W. Hipps, J. Phys. Chem. 104, 11899 (2000)

${ }^{47}$ D. L. Gittins, D. Bethell, D. J. Schiffrin, and R. J. Nichols, Nature (London) 408, 67 (2000).

${ }^{48}$ E. P. Friis, J. E. T. Andersen, L. L. Madsen, P. Møller, and J. Ulstrup, J. Electroanal. Chem. 431, 35 (1997).

${ }^{49}$ E. P. Friis, J. E. T. Andersen, Yu. I. Kharkats, A. M. Kuznetsov, R. J. Nichols, J. Zhang, and J. Ulstrup, Proc. Natl. Acad. Sci. U.S.A. 96, 1379 (1999)

${ }^{50}$ A. M. Kuznetsov and J. Ulstrup, J. Inclusion Phenom. Mol. Recognit. Chem. 35, 45 (1999).

${ }^{51}$ W. Schmickler, in Imaging of Surfaces and Interfaces, edited by J. Lipkowski and P. N. Ross (Wiley, VCH, New York, 1999), p. 305.

${ }^{52}$ R. A. Marcus and N. Sutin, Biochim. Biophys. Acta 811, 265 (1985).

${ }^{53}$ A. M. Kuznetsov, Charge Transfer in Physics, Chemistry, and Biology (Gordon and Breach, Reading, 1995).

${ }^{54}$ A. M. Kuznetsov and J. Ulstrup, Electron Transfer in Chemistry and Biology: An Introduction to the Theory (Wiley, Chichester, 1999). 
${ }^{55}$ A. I. Danilov, Russ. Chem. Rev. 64, 767 (1995).

${ }^{56}$ Nanoscale Probes of the Solid/Liquid Interfaces, edited by A. A. Gewirth and H. Siegenthaler (Kluwer, Dordrecht, 1995).

${ }^{57}$ Imaging of Surfaces and Interfaces, edited by J. Lipkowski and P. N. Ross (Wiley, VCH, New York, 1999).

${ }^{58}$ A. M. Kuznetsov and J. Ulstrup, Probe Microsc. 2, 187 (2001).

${ }^{59}$ A. M. Kuznetsov and J. Ulstrup, J. Electroanal. Chem. 195, 1 (1985).

${ }^{60}$ A. M. Kuznetsov and J. Ulstrup (unpublished).

${ }^{61}$ Yu. Ya. Gurevich and A. M. Kuznetsov, Sov. Phys. Solid State 17, 2076 (1975).

${ }^{62}$ T. X. Lü, J. R. Reimers, M. J. Crossley, and N. S. Hush, J. Phys. Chem. 98, 11878 (1994).

${ }^{63}$ J. R. Reimers, L. E. Hall, M. J. Crossley, and N. S. Hush, J. Phys. Chem. A 103, 4385 (1999).

${ }^{64}$ P. Samori, V. Francke, T. Mangel, K. Müllen, and J. P. Rabe, Opt. Mater. 9, 390 (1998).

${ }^{65}$ M. Menon and D. Srivastava, Phys. Rev. Lett. 79, 4453 (1997).
${ }^{66}$ S. J. Tatis, M. Devoret, H. Dal, A. Thess, R. E. Smalley, L. J. Geeligs, and C. Dekker, Nature (London) 386, 474 (1997).

${ }^{67}$ L. De Cola, V. Balzani, F. Barigelletti, L. Flamigni, P. Belser, A. von Zelewsky, M. Frank, and F. Vögtle, Inorg. Chem. 32, 5228 (1993).

${ }^{68}$ G. S. Hanan, C. R. Arana, J.-M. Lehn, and D. Fenske, Angew. Chem. Int. Ed. Engl. 34, 1122 (1995).

${ }^{69}$ C. Z. Li, H. Sha, and N. J. Tao, Phys. Rev. B 58, 6775 (1998).

${ }^{70}$ C. Z. Li, H. X. He, A. Bogozi, J. S. Bunch, and N. J. Tao, Appl. Phys. Lett. 76, 1333 (2000)

${ }^{71}$ M. S. Kaba, I. K. Song, and M. A. Barteau, J. Vac. Sci. Technol. A 15, 1299 (1997)

${ }^{72}$ M. Fujihira, M. Sakomura, D. Aoki, and A. Koike, Thin Solid Films 273, 168 (1996).

${ }^{73}$ D. Porath, A. Bezryadin, S. de Vries, and C. Dekker, Nature (London) 403, 635 (2000).

${ }^{74}$ A. Broo and M. C. Zerner, Chem. Phys. 196, 423 (1995).

${ }^{75}$ G. J. Ashwell, Thin Solid Films 186, 155 (1990). 